\title{
Abstracts of the 33rd Brain Impairment Conference, May 6-8, 2010, Gold Coast
}

\section{Conference Theme: Innovations in Neurorehabilitation}

\section{PRECONFERENCE WORKSHOPS}

WORKSHOP 1

\section{The Application of Neuropsychological Theory to Clinical Practice}

\author{
Barbara A. Wilson ${ }^{1,2}$
}

1 The Medical Research Council Cognition and Brain Sciences Unit, Cambridge, United Kingdom

2 The Oliver Zangwill Centre for Neuropsychological Rehabilitation, Ely, United Kingdom

\begin{abstract}
Dractising neuropsychologists working in adult brain injury rehabilitation Puse a range of theoretical approaches in their clinical work. In 2002 Wilson published a model of rehabilitation arguing that rehabilitation is one of many fields needing a broad theoretical base incorporating frameworks, theories and models from many different areas. Being constrained by one theoretical model can lead to poor clinical practice. This workshop considers some of the theories and models that have had the most influence on the field of neuropsychological rehabilitation. Included are theories and models of cognitive functioning, learning, assessment, emotion, recovery and plasticity as well as broad based models such as holistic and social models. Results of a survey of the major theoretical models used by British neuropsychologists are presented. We consider how theories and models are employed directly or indirectly in our clinical work. The importance of combining clinical work with research is emphasised. The workshop includes clinical examples and video tapes and concludes with guidelines for good clinical practice all of which have derived from clinical research.
\end{abstract}

\section{WORKSHOP 2}

\section{Using the Australian Therapy Outcome Measures (AusTOMs) to Measure Outcomes Following Brain Impairment}

Carolyn Unsworth ${ }^{1,2,3}$

1 School of Occupational Therapy, La Trobe University, Melbourne, Australia

2 School of Health Sciences, Jokoping University, Sweden

${ }^{3}$ Faculty of Health and Social Care, London South Bank University, London, United Kingdom

Background: The AusTOMs are standardised outcome measures for use by
occupational therapists (AusTOMs for OT), physiotherapists (AusTOMS
for PT) and speech / language therapists (AusTOMs for SP). The AusTOMs
can be used in any practice setting with clients of all ages and all diagnoses,
and takes just a few moments to complete. There are 12 scales for occupa-
tional therapists, 9 for physiotherapists and 6 scales for speech/ language 
therapists. Development of the AusTOMs was made possible through a Commonwealth Government Grant to the Faculty of Health Sciences at La Trobe University. The AusTOMs were developed over a 3 year period with Australian clinicians. AusTOM is based on the UK TOM originally developed by Enderby (1997), and the World Health Organisation's International Classification of Function (WHO, 2001). Over ten articles detailing the reliability, validity and clinical use of these scales have been published. Workshop Aims: (1) explore why we need to measure therapy outcomes, (2) introduce a reliable and valid outcome measure developed in Australia called the Australian Therapy Outcome Measures (AusTOMs) and, (3) practice administering the AusTOMs scales with clients who have brain impairment. Workshop Content: Through attending this workshop, participants will understand the rationale for the development of the AusTOMs, and learn how to reliably administer these measures with clients who have brain impairment. Participants will be shown the generic approach for scoring AusTOMs, including how to select a scale and then measure client outcomes in relation to four domains (Impairment, Activity Limitation, Participation restriction, Distress/Wellbeing). Participants can then practice scoring case study clients using physiotherapy, occupational therapy and speech therapy AusTOMs scales. Finally, the workshop will briefly explore how AusTOMs - OT could be introduced in the participants' workplace. Conclusion: The AusTOMs provide therapists with knowledge of client outcomes that can be used for benchmarking and gathering evidence to support the effectiveness of therapy. It is through using outcome measures such as AusTOMs that we promote best practice in delivering services to clients with acquired brain impairment. An overview of some of the completed and ongoing research in Australia, New Zealand, Sweden, the US and Singapore using AusTOMs will be provided.

\title{
WORKSHOP 3
}

\section{Neurological Vocational Rehabilitation: Assessment and Choices of Placement Model}

\author{
Robert T. Fraser \\ University of Washington, Department of Rehabilitation Medicine - Harbourview Medical Centre, \\ Seattle, Washington, United States of America
}

This presentation begins with a presentation of two levels of vocational
assessment that can be critical to goal setting, a basic and intermediate
level. The basic level of assessment is largely based upon clinical interviews
and psychometric testing. Particular elements of the evaluation process that
will receive attention include vocational interests vis-à-vis work values, syn-
thesising neuropsychological information in vocational planning, assessing
social support, and establishing the need for a community-based 'in vivo'
assessment (intermediate level). The US Department of Labor (1993) waiver
for unpaid work, as a means of providing vocational assessment activity for
people with disability within the community, will be reviewed. The utility of
recommendations as provided through vocational rehabilitation (VR)
providers will also be critiqued and discussed. The second part of the presen-
tation will review 'choice of placement model' using a decision tree format.
There are a number of vocational rehabilitation placement models to be con-
sidered: advisement only, selective brokering, and VR agency vs. company
on-site supports. Each type of these approaches will be discussed in detail to
include the 'paid co-worker as trainer' (under VR agency support). In terms 
of choosing a placement model, key considerations include: client time on the job, neuropsychological and emotional functioning, job complexity, and availability of supervision. This presentation will end with audience participation and response as to placement model choice within the context of different client decision tree variable profiles. The emphasis here is on consistency in approach, but flexibility in placement model use.

\title{
WORKSHOP 4
}

\section{Recent Advances in the Assessment and Treatment of Social Communication Difficulties Following Traumatic Brain Injury}

\author{
Skye McDonald ${ }^{1}$ and Leanne Togher ${ }^{2}$ \\ 1 School of Psychology, University of New South Wales, Australia \\ ${ }^{2}$ Speech Pathology, Faculty of Health Sciences, The University of Sydney, Australia
}

The assessment and treatment of social cognition and everyday discourse abilities is critical to managing the communication problems that can follow traumatic brain injury (TBI). With the increasing pressures on clinicians to facilitate participation goals as part of their treatment planning, there is a need for systematic and theoretically sound ways of measuring everyday interactions. This workshop will explore new ways of measuring discourse skills and social cognition abilities. We discuss the assessment of social skills including social cognition and behaviour and we describe the use of sensitive measures for assessing the nature of discourse and where this breaks down. We also discuss specific techniques for remediating these deficits and review the evidence for their efficacy, with the presentation of recent findings from our own research programs including a trial investigating the effectiveness of training everyday communication partners of people with TBI and a randomised controlled trial of social skills treatment.

\section{PRESIDENTIAL ADDRESS}

\section{Mild Traumatic Brain Injury in Older Adults: Cause for Concern?}

Glynda Kinsella ${ }^{1,2}$

${ }^{1}$ Psychological Science, Latrobe University, Melbourne, Australia

2 Psychology Department, Caulfield Hospital, Melbourne, Australia

\begin{abstract}
A $\mathrm{n}$ increasingly important demographic group presenting to emergency and trauma services are older adults (65 years+) who experience traumatic brain injury (TBI) as a result of a fall or a motor vehicle accident. Approximately $60 \%$ of all TBI episodes are classified as mild and, encouragingly, in young adults a generally good outcome is expected by 3 -months post-injury. This positive outcome has not been established for older adults who can be considered to be at risk of persisting difficulties in consequence of a range of risk factors. The factors affecting early recovery for older adults will be reviewed and data will be presented from our ongoing study of outcome for older adults following mild TBI.
\end{abstract}


KEYNOTE AND INVITED SPEAKERS

INTERNATIONAL KEYNOTE ADDRESS 1

\title{
Cutting Edge Developments in Neurorehabilitation and Future Directions
}

\author{
Barbara A. Wilson ${ }^{1,2}$
}

1 The Medical Research Council Cognition and Brain Sciences Unit, Cambridge, United Kingdom

2 The Oliver Zangwill Centre for Neuropsychological Rehabilitation, Ely, United Kingdom

\begin{abstract}
Teuropsychological rehabilitation is concerned with the amelioration of 1 cognitive, emotional, psychosocial and behavioural deficits caused by an insult to the brain. Major changes have occurred over the past decade or so. Neurorehabilitation now tends to be seen as a partnership between survivors of brain injury, their families and professional staff who negotiate and select meaningful goals to be achieved. There is a widespread recognition that cognition, emotion and psychosocial functioning are interlinked and all should be targeted in rehabilitation. This is the basis of the holistic approach. There is increasing use of technology to compensate for cognitive deficits and there is a greater understanding that rehabilitation needs to draw on a number of theories, models and frameworks to address the many and complex consequences of brain injury. This presentation looks at some of the most recent developments in neuropsychological rehabilitation including (a) new assessment procedures (b) new treatment strategies for cognitive, emotional and psychosocial problems (c) new technology to help people compensate for their everyday problems and (d) new theoretical models to improve our understanding of the consequences of brain injury. The final part of the presentation considers possible future developments in rehabilitation. We consider stronger links with basic neuroscience, better use of imaging procedures, collaboration with pharmaceutical companies, better evaluation of our programs and look at the possibility of brain-computer interaction methods.
\end{abstract}

\section{INTERNATIONAL KEYNOTE ADDRESS 2}

\section{Neurological Vocational Rehabilitation: State of the Science}

Robert T. Fraser

University of Washington, Department of Rehabilitation Medicine - Harbourview Medical Centre, Seattle, Washington, United States of America

$\mathrm{T}$ This presentation reviews the state of the science related to work access/work return for individuals with neurological disability. Initial studies are reviewed related to the risk of unemployment related to the general population, the history of job stability post-work return, and capacity for handling job complexity. Predictors of work return and job proficiency are also highlighted. The mid part of the presentation emphasises vocational rehabilitation (VR) outcomes with intervention as related to US TBI Model Systems and other intervention studies - the current emphasis being upon establishing elements of the VR intervention that are most effective. Barriers to vocational success are also quantified by rehabilitation personnel and presented for review. Issues relating to limited VR interventional research are also highlighted. Concluding remarks relate to clinical enhancers/stabilising 
influences relating to work access/work return. The type of partnership and specialisation needed for VR success and the field of vocational rehabilitation is also reviewed in detail.

\title{
NATIONAL KEYNOTE ADDRESS 1
}

\section{Mapping the Neural Substrates of Naming Facilitation in Aphasia: Preliminary Findings}

\author{
David Copland, ${ }^{1,2}$ Shiree Heath, ${ }^{2}$ Katie McMahon, ${ }^{3}$ Lyndsey Nickels, ${ }^{4}$ \\ Anthony Angwin, ${ }^{1}$ Anna Holmes, ${ }^{2}$ Greig de Zubicaray, ${ }^{3}$ Erin Smith, ${ }^{1}$ Elizabeth Cardell \\ 1 School of Health and Rehabilitation Sciences, The University of Queensland, Australia \\ 2 Centre for Clinical Research, The University of Queensland, Australia \\ ${ }^{3}$ Centre for Magnetic Resonance, The University of Queensland, Australia \\ ${ }^{4}$ Macquarie Centre for Cognitive Science, Australia
}

\begin{abstract}
Tntroduction: The neural substrates of naming facilitation in aphasia are currently unknown. Event-related functional MRI with overt naming production provides an unprecedented opportunity for addressing this issue. Methods: S1, a 37-year-old female with aphasia 11 years post stroke (involving a left fronto-temporal lesion) participated in the study. At the time of the initial study, S1 obtained an Aphasia Quotient of 88 on the Western Aphasia Battery and 43/60 on the Boston Naming Test. Two imaging studies were conducted examining brain activity (1) pre and post blocks of semantic versus phonological naming treatment and (2) following brief periods of naming facilitation. Images were acquired with sparse acquisition on a Bruker 4 Tesla MRI scanner. Items to be treated were selected from unnamed items during the pre-treatment scan, and from a large naming corpus for the followup facilitation study. Results: Behavioural testing indicated improvements in naming following both semantic and phonological treatments. Imaging data indicated increased activity in perilesional and left temporal regions during naming of items treated with a semantic approach. The phonological treatment was associated with a different pattern of activity involving right posterior regions not associated with language. Discussion: These findings suggest that in addition to recruiting perilesional regions, naming treatments based on a specific level of the naming process (e.g., semantics) may engage left hemisphere mechanisms normally involved in that process, but this pattern may vary depending on the process targeted. Comparisons between intense therapy over several weeks and short-term naming facilitation will also be considered.
\end{abstract}

\section{NATIONAL KEYNOTE ADDRESS 2}

\section{Innovations and Challenges in Driver Assessment and Rehabilitation}

Carolyn Unsworth

School of Occupational Therapy, Latrobe University, Australia

$\mathrm{T}$ The occupation of driving provides more than community mobility. The ability to drive is seen as a rite of passage to adulthood and is valued for the independence and freedom it provides. However, participating in driving may be challenged by the physical, cognitive, perceptual and sensory problems that can accompany acquired brain impairment. People with brain impairment who have never driven and wish to learn, and experienced 
drivers who wish to resume driving following brain impairment, require assessment to determine fitness-to-drive. In many states in Australia, and internationally, occupational therapists are involved in these assessments. This paper explores some of the issues surrounding driver assessment and rehabilitation, and commences by describing the process of driver assessment and rehabilitation for people with brain impairment. The paper then focuses on the off-road assessment and presents the rationale for development of a new standardised assessment called the Occupational Therapy Driver Off-Road Assessment (OT-DORA) battery. Research evidence to support components of the OT-DORA is presented including a study examining which test of road law and road craft knowledge to include in the battery as well as information on the OT Drive Home Maze Test which shows excellent predictive validity for on-road driving performance. The development of the OT-DORA Battery supports occupational therapists' ability to accurately and consistently assess and report the driving capacity of clients to medical staff and/ or licensing authorities. This helps ensure our roads are as safe as possible for all road users.

\title{
NATIONAL KEYNOTE ADDRESS 3 \\ The Prevalence, Course, Comorbidities and Burden of Challenging Behaviours After Traumatic Brain Injury: The Experience of the NSW Brain Injury Rehabilitation Program
}

\author{
Grahame Simpson ${ }^{1,2}$ on behalf of the \\ NSW GMCT Brain Injury Directorate Challenging Behaviours Project Group \\ ${ }^{1}$ Brain Injury Rehabilitation Unit, Liverpool Hospital, Australia \\ 2 Rehabilitation Studies Unit, University of Sydney, Australia
}

Tntroduction: Challenging behaviours after traumatic brain injury (TBI) were identified as a clinical priority issue by the NSW Brain Injury Rehabilitation Program (BIRP). Challenging behaviours were defined as encompassing physical and verbal aggression, inappropriate sexual behaviour, perseveration, wandering/absconding, inappropriate social behaviour and adynamia. All 11 adult BIRP units participated in the project to produce a state-wide plan aimed at improving the management of these behaviours. Methods: All active adult community-based clients of the NSW BIRP who had a TBI $(n=659)$ were rated by their prime treating clinician on standardised measures of challenging behaviour, function, comorbidity, participation and support needs. Additional data were collected on current service utilisation and service delivery variables. Three month follow-up data were gathered for a subgroup $(n=324)$. Finally, in-depth case studies were conducted on 28 clients nominated by the BIRP services as their 'most challenging' cases. Results: Clients had predominantly severe injuries $(6 \%$ mild-moderate, $12 \%$ unknown) and 53\% displayed behaviours classified as challenging with inappropriate social behaviour and verbal aggression most frequent. Severe alcohol and psychiatric conditions were uncommon. Discussion: Data were integrated to form the basis for the state plan. Key recommendations address improvements in staff training, coordination between relevant state agencies, family training and support as well as increased resources. 


\title{
INTERNATIONAL PLENARY ADDRESS
}

\section{Is There a Case for Supporting Resiliency-Based Interventions in Children Following Acquired Brain Injury?}

\author{
James Tonks, ${ }^{1}$ Philip J. Yates ${ }^{1,2}$ and W. Huw Williams ${ }^{1}$ \\ ${ }^{1}$ Centre for Clinical Neuropsychological Research, School of Psychology, University of Exeter, \\ United Kingdom \\ ${ }^{2}$ Department of Psychological Medicine, Devon Partnership NHS Trust, United Kingdom
}

\begin{abstract}
Tntroduction: Preliminary studies with healthy children indicate that individual factors associated with executive function (EF) (specifically self and emotional regulation) are important individual attributes of resilience and can influence psycho-social functioning, although no studies of children with brain injury, or indeed any neurological impairment, have investigated this assumption. The researchers were particularly interested in the potential mediating impact of resilience on outcomes and aimed to explore the relationship between resilience, socio-emotional behaviour, distress and executive function. Aims: Whether resilience is influential in mediating relationships between executive dysfunction and socio-emotional behaviour disturbance after TBI. Whether there is deterioration in a young person's sense of resilience associated with the emergence of EF difficulties after TBI when compared to healthy controls. Methods: The study will use a within and between subjects design. ABI participants will be aged between 9 and 17 years with head injuries. These will be compared to a group of agematched matched non-injured controls. A range of hypotheses will be tested to determine differences between the healthy and ABI groups whilst controlling for socio-economic status and family functioning. The impact of resiliency as a predictor of better socio-emotional behavioural outcome in the two groups will be investigated, as well as whether resiliency, or traits of resiliency, mediate(s) the relationship between EF and socio-emotional behaviour. Results and Discussion: Preliminary findings from this study regarding the potential mediating impact of resilience will be presented, and the implications for intervention and rehabilitation will be discussed.
\end{abstract}

\section{FRIDAY MAY 7}

\section{CONCURRENT SESSION 2: OUTCOMES AND MEASUREMENT}

\section{The Relationship Between Apoe Genetic Status and Outcome After Traumatic Brain Injury}

\author{
Jennie Ponsford, ${ }^{1,2}$ Anna McLaren, ${ }^{1}$ Dion Ruzki, ${ }^{1}$ Michael Schonberger, ${ }^{1,2}$ \\ John Olver ${ }^{1,2}$ and Michael Ponsford ${ }^{2}$ \\ 1 School of Psychology and Psychiatry, Monash University, Australia \\ 2 Monash-Epworth Rehabilitation Research Centre, Epworth Hospital, Melbourne, Australia
}

\begin{abstract}
Tntroduction: In recent years there has been growing interest in the role of 1 the Apolipoprotein (ApoE) gene in influencing outcome following TBI. ApoE plays a role in cell maintenance and nervous system response to injury. Its three isoforms, $\varepsilon 2, \varepsilon 3$ and $\varepsilon 4$, show differing responses to brain injury; with $\varepsilon 4$ allele carriers potentially having poorer recovery. Some previous studies have found poorer outcomes in $\varepsilon 4$ allele carriers, but others have not. Most have had limited statistical power. The current study examined whether presence of the ApoE $\varepsilon 4$ allele was associated with lower
\end{abstract}


Glasgow Coma Scores (GCS), longer post-traumatic amnesia (PTA) duration and poorer long-term functional outcome. Methods: Participants were patients attending a rehabilitation centre followed up 1-5 years post-injury. They comprised 654 individuals with TBI (67.4\% male). Functional outcome was measured on the Glasgow Outcome Scale — Extended (GOSE). ApoE genotyping was determined from saliva samples by one-stage PCR method. Results: The ApoE $\varepsilon 4$ allele was carried by 166 (25.3\%) participants, most having the $3 / 4$ allele combination. Of non-ApoE $\varepsilon 4$ carriers, most had the $3 / 3$ combination. Only three participants had the $2 / 2$ combination. The GOSE was completed a mean of 1.9 years post-injury $(S D=1.3)$. We found the hypothesised relationship between ApoE $\varepsilon$ status and functional outcome on the GOSE. There was no significant relationship between initial injury severity, measured by GCS or PTA duration and genetic status. Discussion: It appears that presence of the ApoE e4 allele may influence long-term functional outcome. Possible mechanisms underpinning this relationship will be discussed.

\title{
Prediction of Psychosocial Outcome One Year After Traumatic Brain Injury: A Structural Equation Modelling Approach
}

\author{
Michael Schönberger ${ }^{1,2}$ and Jennie Ponsford ${ }^{1,2,3}$ \\ 1 School of Psychology, Psychiatry and Psychological Medicine, Monash University, Australia \\ 2 Monash-Epworth Rehabilitation Research Centre, Epworth Hospital, Melbourne, Australia \\ ${ }^{3}$ National Trauma Research Institute, Melbourne, Australia
}

Introduction: The purpose of the present study was (1) to test and optimise a comprehensive model of the prediction of functional as well as employment outcome after TBI, and (2) to compare this model between those employed and unemployed, respectively, at the time of injury. Method: Participants: $N=949$ individuals ( $74 \%$ males; median age at injury 26 years; median duration of PTA 17 days) who had been admitted to Epworth Hospital, Melbourne for rehabilitation after TBI. Outcome 1 year post injury: Employment status and experience of post-injury cognitive, behavioural and emotional changes. Predictors: gender, age, education, preinjury employment status, pre-injury mental disorder, duration of PTA, orthopaedic injuries. A Structural Equation Model (SEM), based on the existing literature, was developed, tested, and modified. This SEM also modelled the inter-relationships between the predictors. Results: The fit of a modified SEM was good (CFI=.99; RMSEA=.03, however, $\chi^{2}(59)=$ 103.37, $p<.001)$. All outcome variables were significantly related to each other. As hypothesised, both direct and mediated relationships between predictor and outcome variables were found, with PTA being the strongest predictor of outcome. Although overall, the pattern of predictors did not depend on employment status at the time of injury, the predictive power of the model tended to be higher in those unemployed at the time of injury. Discussion and Conclusion: The results of this study improve our understanding of the complex inter-relationships between the predictors and the different aspects of outcome after TBI. Further research differentiating between different subgroups of the TBI population is needed. 


\title{
The Functional Independence Measure for People With Traumatic Brain Injury: How Good is the Cognitive Scale for Measuring Neuropsychological Impairments?
}

\author{
Robyn L. Tate, ${ }^{1,2}$ Natasha Lannin,, ${ }^{1,2}$ Christine Taylor ${ }^{2}$, Anne Pfaff, ${ }^{3}$ Donna Wakim, ${ }^{1}$ \\ Pamela David, ${ }^{1}$ Bronwyn Coward ${ }^{1}$ and Carmel Whitty ${ }^{4}$ \\ ${ }^{1}$ Rehabilitation Studies Unit, Northern Clinical School, Sydney, Medical School, \\ University of Sydney, Australia \\ 2 Brain Injury Unit, Royal Rehabilitation Centre, Sydney, Australia \\ ${ }^{3}$ Brain Injury Rehabilitation Unit, Liverpool Hospital, Sydney, Australia \\ ${ }^{4}$ Private Practice, Sydney, Australia
}

\begin{abstract}
Rackground: The Functional Independence Measure (FIM) is one of the

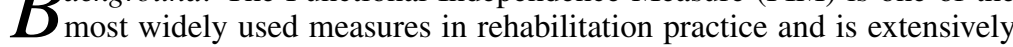
used in research. Although there is substantial psychometric evidence to support the validity of the Motor Scale of the FIM, surprisingly little empirical data are available for the Cognitive Scale. Aim: to examine the criterion and construct validity of the Cognitive Scale of the FIM. Method: Sixty people with traumatic brain injury from inpatient rehabilitation $(n=28)$ and community outreach $(n=32)$ programs in Sydney were administered the FIM and other measures. Validating instruments were selected to specifically target each of the five FIM cognitive items, and included both objective cognitive tests and relative report using standardised questionnaires. Results: Correlation coefficients between similar constructs were, at best, modest. Coefficients for objective cognitive tests ranged between $r=0.28$ (FIM-Comprehension vs. Measure of Cognitive Linguistic Abilities Receptive score) and $r=0.55$ (FIM-Memory vs. Rey Auditory Verbal Learning Test). Somewhat higher coefficients were found for the questionnaire data, ranging from $\mathrm{r}=-0.60$ (FIM-Comprehension vs. La Trobe Communication Questionnaire (LCQ); FIM-Social Interaction vs. Frontal Systems Behaviour Scale - Disinhibition) to $r=-0.81$ (FIM-Expression vs. LCQ). No FIM cognitive items discriminated between subgroups stratified by injury severity. Conclusions: These results raise questions about the criterion and construct validity of the Cognitive Scale of the FIM for people with traumatic brain injury. Further critical evaluation of the applicability of this tool in assessing the complex neuropsychological deficits found in the TBI population is needed.
\end{abstract}

\section{There is More to the Functional Independence Measure Than Meets the Eye: A Critical Analysis of FIM Administration Procedures}

Donna Wakim, ${ }^{1}$ Robyn Tate, ${ }^{1,2}$ Natasha Lannin, ${ }^{1,2}$ Anne Pfaff $^{3}$ and Christine Taylor ${ }^{2}$

${ }^{1}$ Rehabilitation Studies Unit, Northern Clinical School, University of Sydney, Australia

2 Brain Injury Unit, Royal Rehabilitation Centre, Sydney, Australia

${ }^{3}$ Brain Injury Rehabilitation Unit, Liverpool Hospital, Sydney, Australia

The 18-item Functional Independence Measure (FIM) is widely used in rehabilitation as a clinical indicator; in fact, the Australian Council on Healthcare Standards requires that the FIM be scored within 72 hours of admission to hospital for rehabilitation and again within 72 hours of discharge. In rehabilitation settings, the FIM is often scored in the context of a case conference in which professionals from different disciplines rate those items which match their area of expertise (eg., speech pathologists for the two communication items). This raises the issue as to whether the FIM is being rated in its own right or whether it is benefiting from results of other assessments that have been conducted. The present study is part of a broader 
project validating the cognitive scale of the FIM. It reports on preparatory work undertaken on the FIM to ensure that the independent FIM assessor remained blind to other sources of information. In order to do this, a series of closed questions was developed for each FIM item, based on the decisionmaking trees used to score the FIM. The questions were then asked of clinical staff who had not performed any objective testing with the person or with a family member. In this presentation, the development of the questions asked to score the FIM will be outlined, as well as the issues confronted in scoring the FIM as a blinded assessor.

\section{Sleep Disturbance and Melatonin Levels Following Traumatic Brain Injury}

Jennie L. Ponsford, ${ }^{1,2}$ Julia A. Shekleton, ${ }^{1}$ Diane L. Parcell, ${ }^{1}$ Jennifier R. Redman ${ }^{1}$, Jo Phipps-Nelson ${ }^{1}$ and Shantha M. W. Rajaratnam ${ }^{1}$

1 School of Psychology And Psychiatry, Monash University, Australia

2 Monash-Epworth Rehabilitaiton Research Centre, Epworth Hospital, Melbourne, Australia.

Tntroduction: Sleep disturbances commonly follow traumatic brain injury (TBI), impact on rehabilitation and contribute to ongoing disability. However, there are no conclusive findings regarding changes to sleep quality and sleep architecture in TBI patients. Possible causes of sleep disturbances include disruption of the circadian regulation of sleep-wake cycles, psychological distress and/or neuronal response to injury. This study investigated possible underlying mechanisms of sleep-wake disturbance following TBI. Methods: 23 TBI patients (429.7 \pm 287.62 days post injury) and 23 age- and gender-matched controls were compared on polysomnographic sleep parameters, salivary dim light melatonin onset (DLMO) time and selfreported levels of sleep quality, anxiety and depression. Results: TBI patients reported significantly more anxiety and depressive symptoms and sleep disturbance than controls. Polysomnography showed significantly decreased sleep efficiency (SE) and increased wake after sleep onset (WASO) in the TBI group. Although no significant group differences were found in sleep architecture, when anxiety and depression scores were controlled, TBI patients showed higher amount of slow wave sleep. No differences in self reported sleep timing or salivary DLMO time were found. However, TBI patients showed significantly lower levels of evening melatonin production. Melatonin level was significantly correlated with REM sleep but not SE or WASO. Conclusions: Reduced evening melatonin production may indicate disruption to the circadian regulation of melatonin synthesis. The results suggest that at least two factors contribute to sleep disturbances in TBI patients: that elevated depression is associated with reduced sleep quality, and increased slow wave sleep is attributable to effects of mechanical brain damage. 


\section{CONCURRENT SESSION 3: SOCIO-COGNITIVE OUTCOMES}

\section{Making Sense of Self in Alzheimer's Disease: Reflective Function and Memory}

Louise A. Simm, ${ }^{1}$ Robert D. Jamieson, ${ }^{1}$ Glynda J. Kinsella, ${ }^{1}$ Ben Ong ${ }^{1}$ and Mark W. J. Garner ${ }^{2}$

${ }^{1}$ La Trobe University, Australia

2 University of Aberdeen, United Kingdom

$\mathrm{T}$ The effect of Alzheimer's disease on maintaining a sense of self may be complicated by the memory impairment and cognitive decline associated with the illness. One vehicle for measuring sense of self is with theory of mind, the ability to attribute mental states, including thoughts and feelings, to oneself and others. A modified version of the Reflective Function Scale (originally developed by Fonagy, Steele, Steele, Moran, \& Higgitt, 1991), which measures capacity for assessing mental states in self and others, provided a rating for theory of mind. The current study explored the relationship between reflective function, episodic memory and working memory in 49 participants with mild Alzheimer's disease and 26 healthy age-matched control participants in semi-rural Victoria, Australia. The mild Alzheimer's disease sample had a lower personal reflective function and social reflective function than the age-matched control sample. Delayed Free Recall, an indicator of episodic memory, predicted personal reflective function for the Alzheimer's disease sample, but not the control sample. While further research is necessary, this is the first study that has explored the impact of Alzheimer's disease on reflective function, and the findings suggest that sense of self (personal reflective function) does diminish with the development of Alzheimer's disease.

\section{Identifying Impairments in Social Functioning Using a Novel Task}

Michelle A. Kelly, ${ }^{1}$ Skye McDonald, ${ }^{1}$ Jacqueline Rushby ${ }^{1}$ and David Kellett ${ }^{2}$

1 School of Psychology, University of New South Wales, Australia

2 Hunter Brain Injury Service, Newcastle, Australia

$\mathrm{D}$ ecision-making forms an important part of our everyday lives and is commonly impaired after a severe traumatic brain injury (TBI). This study aimed to develop and pilot a social decision making task, and examine the electrodermal and heart rate activity associated with good and bad social choices. Participants play a game of 'catch and throw' with four pseudo players over the Internet. Two pseudo players are regarded 'good choices' as they return the ball at a probability of $60 \%$ or $30 \%$; the other two players are 'bad choices', returning the ball at a probability of $10 \%$ or $0 \%$. Behavioural data are presented for 18 adults with severe TBI (PTA $M=59.3$ days), aged between 24 and 66 years $(M=45.23)$ tested at least 8 months post-injury ( $M$ $=5.5$ years). These were compared to 23 control participants (10 matched, 13 pilot data). Preliminary results indicate that control participants made better decisions than TBI participants, thus, winning more throws overall ( $t$ $=2.13, p=.04$ ). A significant interaction between group and choice type was observed with control participants making more good choices and less bad choices than the TBI group $(F=13.25, p=.001)$. Psychophysiological data was collected for 12 control participants. Preliminary results indicate that heart rate is highest and skin conductance lowest prior to making a good choice. This novel task may help elucidate the nature of poor social decisionmaking in TBI patients. 


\title{
The Assessment of Interpersonal Communication Skills in Stroke Patients
}

Margaret Turner and David Andrewes

School of Behavioural Science, The University of Melbourne, Australia

\begin{abstract}
$I^{2}$ mpaired interpersonal communication skills and related depression are common after a stroke. Suitable measures of emotional processing appropriate for assessing therapeutic gain following psychotherapy for these patients, however, are not available. To address this deficiency a verbal and a non-verbal version of an emotion in faces task to assess static emotion recognition and two versions of a videoed common interpersonal situation scenario task to assess dynamic emotional recognition, understanding and appropriate response were therefore developed. These tasks were then used to investigate the state of emotional processing in 25 stroke patients and 31 rheumatic/orthopaedic control patients. Participants were randomly assigned to either the verbal or the non-verbal emotion in faces task and one of the versions of the emotional scenarios task. Related cognitive and emotional processing deficits were also assessed to determine their influence on emotional processing. Deficits in emotional processing for the stroke patients compared to the controls were identified. Right hemisphere brain damage was found to significantly impair emotional perception and emotional understanding and depression was found to significantly impair the ability to respond to an emotional situation in an appropriate manner. There was no relationship between emotional processing and deficits in cognitive and emotional resources, however a lack of insight in right hemisphere brain damaged patients may interfere with the results. These findings demonstrate that both tasks may be suitable measures of emotional processing in stroke patients and indeed all brain-damaged patients and that they could successfully be incorporated into psychotherapy for post stroke depression.
\end{abstract}

\section{Thinking Strategically: An Innovative Approach to Neurorehabilitation}

\author{
Melissa Nott ${ }^{1,2}$ and Christine Chapparo ${ }^{2}$ \\ 1 Westmead Hospital, Brain Injury Rehabilitation Service, Sydney, Australia \\ 2 The University of Sydney, Faculty of Health Sciences, Australia
}

ackground: Rehabilitation approaches based on cognitive strategy
instruction are being increasingly used in adult brain injury rehabilita-
tion. Cognitive strategies are used during occupational performance to
enhance perception of a task in the context of the environment, recognition
of similarities between the present task and previous experiences, identifica-
tion of relevant task cues, enhancement of goal formation, and decision
making about performance. Research design: Single case studies comparing
current occupational therapy (OT) intervention with the Perceive, Recall,
Plan and Perform (PRPP) System of Intervention. Intervention approach:
The PRPP System of Intervention is a strategy instruction based approach
used by occupational therapists to facilitate every-day functional perfor-
mance through targeting of specific information processing strategies that
are applied during task performance. The PRPP System of Intervention
focused on information processing strategies to structure and organise infor-
mation required for effective task performance, focusing attention, high-
lighting integral task components, facilitating recall of task procedures,
identifying and avoiding obstacles to performance, use of internally driven
tactics, and goal direction. Findings: Participants significantly improved in
their application of processing strategies following PRPP Intervention in 
comparison to current OT Intervention. Several features of the PRPP Intervention approach were hypothesised to underpin the level of success achieved. Cognitive strategies were 'chunked' together to enable instruction of multiple strategies described as 'Stop, Attend, Sense, Think, Do' to better facilitate flow of information processing. The PRPP Intervention focused on 'thinking strategies' rather than 'doing strategies'. 'Bridging' techniques were used by the therapist to facilitate generalisation of cognitive strategies to multiple functional tasks and settings.

\title{
Autonomic Responses To Facial Expressions Following Severe Traumatic Brain Injury
}

Jacqueline Rushby, ${ }^{1}$ Skye McDonald, ${ }^{1}$ Sophie Li, ${ }^{1}$ Arielle Desousa, ${ }^{1}$ Aneta Dimoska, ${ }^{1}$ Charlotte James ${ }^{1}$ and Robyn Tate ${ }^{2}$

1 School of Psychology, University of New South Wales, Australia

${ }^{2}$ Rehabilitation Studies Unit, Department of Medicine, Sydney University, Australia

Drevious research has found that people with traumatic brain injury (TBI) display abnormal affective responses when passively viewing emotionally charged material, with negative emotions more frequently impaired than positive. The neuropsychological mechanisms underpinning such deficits are as yet unclear. This study examined whether emotion perception deficits can be improved by focusing attention. Eighteen adults with moderate-tosevere TBI and 18 control participants viewed facial expressions from the Ekman and Friesen series while their autonomic responses (skin conductance (SCR) and heart rate (HR)) were monitored. Each individual was presented with two blocks of faces ( 8 angry and 8 happy per block). Participants passively viewed the faces for block one, and were instructed to identify the emotional expression for block two. For the passive task, control participants demonstrated larger SCRs and an increase in HR acceleration when viewing angry expressions relative to happy, whereas no differences were shown between expressions for the TBI group. A significant emotion $\mathrm{x}$ group interaction emerged in the absence of either emotion or group main effects for each measure (SCR: $F=4.51, p=.041$; HR: $F=4.52, p=.041$ )). Instructions to focus attention upon the emotional content of the faces produced a markedly different response profile for HR. Responses were larger for controls than TBI participants $(F=9.22, p=.005)$, and enhanced to angry compared with happy expressions for both groups $(F=8.53, p=.006)$. The results support the view that increasing attention facilitates emotion recognition in people with TBI.

\section{Exploring The Mechanisms Behind Poor Emotion Perception In People With Severe Traumatic Brain Injury: The Role of Mimicry}

\author{
Skye McDonald, ${ }^{1}$ Sophie Li, ${ }^{1}$ Arielle De Sousa, ${ }^{1}$ Jaqueline Rushby, ${ }^{1}$ Charlotte James ${ }^{1}$ \\ and Robyn Tate ${ }^{2}$ \\ 1 School of Psychology, University of New South Wales, Australia \\ 2 Rehabilitation Studies Unit, Department of Medicine, University of Sydney, Australia
}

Introduction: It is now well established that many individuals with severe
traumatic brain injury (TBI) do not recognise emotional expressions nor-
mally, especially negative emotions. The neuropsychological mechanisms are
yet to be elucidated. In this study we examined whether automatic facial
mimicry to expressions is impaired in people with TBI and whether this
relates to accuracy in emotion recognition. Methods: Twenty-one adults with 
severe TBI and 20 control participants viewed angry and happy facial expression. Facial movement of the Corrugator supercilii (brow) and Zygomaticus major (cheek) was monitored using EMG. Participants were also assessed for their ability to identify emotional expressions. Results: The control group demonstrated increased brow activity to angry faces and increased cheek activity to happy faces. The TBI group showed mimicry to happy faces only. Facial mimicry did not correlate with emotion perception accuracy for either group. Conclusion: These results add to growing evidence for impaired automatic responses to emotion in people with TBI. The results did not support the view that simulation (mimicry) facilitates emotion recognition.

\title{
CONCURRENT SESSION 4: FUNCTIONAL OUTCOMES AND INTERVENTIONS Participant Perceptions of a Communication Training Program for People With Traumatic Brain Injury and Their Communication Partners
}

\author{
Leanne Togher, ${ }^{1}$ Emma Power, ${ }^{1}$ Skye McDonald, ${ }^{2}$ Robyn Tate ${ }^{3,4}$ and Rachael Rietdijk ${ }^{1,5}$ \\ 1 Speech Pathology, Faculty of Health Sciences, The University of Sydney, Australia \\ 2 School of Psychology, The University of New South Wales, Australia \\ ${ }^{3}$ Rehabilitation Studies Unit, Northern Clinical School, Faculty of Medicine, University of Sydney, Australia \\ ${ }^{4}$ Royal Rehabilitation Centre, Sydney, Australia \\ ${ }^{5}$ Brain Injury Rehabilitation Unit, Liverpool Health Service, Sydney, Australia
}

ntroduction: Recently, communication partner training programs have
emerged as an alternative method of improving communication skills of people with traumatic brain injury (TBI). Communication problems following TBI can contribute to socially inappropriate behaviours and poor psychosocial outcomes. Two treatments have been shown to improve communication in people with TBI: (1) social skills training for the person with TBI alone and (2) training communication partners to deal with difficult communication behaviours. A recently completed clinical trial that included communication partners has shown statistically significant improvements in interactions of people with TBI using objective measures. However, it is also critical to consider the participants' perceptions of the training process. This research aims to describe, and examine participant perceptions of their experience with a communication skills training program that was part of a controlled clinical trial (Togher et al., 2008). Method: Participants included 14 people with severe TBI and their everyday communication partners (ECPs) who completed a 10-week communication partnertraining program. Each pair participated in a videotaped, structured interview that was later transcribed. Questions focused on the participants' experiences throughout the training process and asked for suggestions for the development of future training programs. The transcripts were analysed for themes using constant comparative analysis techniques (Glaser \& Strauss, 1967). Results: Thematic analysis revealed four overarching themes. Two themes focused on participants' perceived benefits of the training. Both participants with TBI and their ECPs felt that (1) the training program improved their communication skills and (2) these better skills led to improvements in other areas of their life. The two remaining themes related to participants' experience with the training process itself. Participants with TBI and their ECP's indicated that there were (1) key elements of the program that were important to their success, for example, the importance of the inclusion of communication partners as the key focus of the training program. However, participants also identified (2) challenges they experienced throughout the program. For some communication partners, the focus on 
their own communication behaviours was initially confronting. Participants provided suggestions for modifications to the program that may have further enhanced their success. Discussion: While quantitative data is essential to determine the efficacy of a clinical trial, it is also important to consider the participants' experiences of the process. Conducting detailed interviews provided information regarding consumer satisfaction, potential strengths and changes to the program and social validation data to compliment quantitative data. Modifying communication behavior is a complex and challenging area of clinical research and the results of these interviews confirmed that, while difficult, it can be life changing.

\title{
The Accuracy and Feasibility of GPs Devices for Measuring Community Participation
}

\author{
Annie McCluskey, ${ }^{1,2,3}$ Louise Ada, ${ }^{4}$ Catherine Dean ${ }^{4}$ and Janine Vargas ${ }^{4}$ \\ ${ }^{1}$ Community Based Health Care Research Unit, Faculty of Health Sciences, \\ The University of Sydney, Australia \\ 2 The Royal Rehabilitation Centre, Sydney, Australia \\ ${ }^{3}$ The National Stroke Foundation, Australia \\ ${ }^{4}$ Discipline of Physiotherapy, Faculty of Health Sciences, The University of Sydney, Australia
}

Tntroduction: Self-report methods such as diaries are typically used to meaI sure outdoor journeys and community participation. However, they are burdensome and often produce unreliable or inaccurate data. Direct observation is considered the 'gold standard' measure but it is time consuming and costly. Our aims were to investigate (a) the feasibility of people with stroke using a global positioning system (GPS) device, and (b) the accuracy of the device to record their outdoor journeys. Methods: Communitydwelling people $(n=20)$ post-stroke who could walk independently carried a GPS device for 7 consecutive days that recorded where they travelled, distance travelled, speed travelled and the number of outings. Diaries were also kept for these 7 days. On Day 2, participants were observed for four hours. Descriptive statistics will be used to analyse feasibility data (e.g., compliance, preferences). Intraclass correlation and the kappa statistic will be used for data analysis to compare agreement between the GPS device, self-report diaries and the 'gold standard' measure (direct observation). Results: Data collection will be complete by early 2010 . Results will include acceptability by participants; feasibility of recruitment (consent/decline rate); feasibility of the GPS device (e.g., battery life, recharging, device failure); sensitivity and specificity of the GPS device and self-report diary when compared to observation. Discussion: If feasible and accurate, the GPS device represents an objective measure of community participation for use in future clinical trials. Subsequent studies will be required with other groups such as people with traumatic brain injury, intellectual disability, older adults, children and wheelchair users. 


\title{
Goal Directed, Outpatient Rehabilitation for Adults With Traumatic Brain Injury: Comparison of Outcomes in Home and Day Hospital Settings
}

\author{
Emmah Doig, ${ }^{1}$ Jennifer Fleming, Petrea Cornwell ${ }^{1,2}$ and Pim Kuipers ${ }^{3}$ \\ 1 The University of Queensland, School of Health and Rehabilitation Sciences, Australia \\ 2 Princess Alexandra Hospital, Brisbane, Australia \\ ${ }^{3}$ Centre for Remote Health, A Joint Centre of Flinders University and Charles Darwin University, Australia
}

\begin{abstract}
Tntroduction: Outpatient neurological rehabilitation is delivered in a variety of settings including day hospitals, outpatient clinics and in homes. Whilst there is an increasing trend towards delivery of home-based rehabilitation, according to the findings of a systematic review, there have been no previous controlled studies designed to investigate the influence of therapy context on outcomes for people with TBI receiving outpatient neurological rehabilitation. Method: A controlled, repeated measures, crossover design with pre, and post-test measures was used to compare clinical change following a (A) home-based treatment phase and a (B) day hospital-based treatment phase of a goal-based outpatient rehabilitation program for a group of 14 participants with TBI. To counterbalance order effects, participants were randomly allocated to a treatment sequence $\mathrm{AB}$ or $\mathrm{BA}$ and all outcome assessments were conducted by a researcher not involved in treatment and blinded to the treatment sequence. The rehabilitation experience in both settings was explored using semi-structured interviews with the participants, their significant others and the treating occupational therapists. Results: Participants were significantly more satisfied with therapy at home. There was a trend towards better outcomes on all other measures post the home phase however the differences in gains following home and day hospital phases were not statistically significant. The main emerging qualitative theme was that the perceived culture of hospital and boundaries create restrictions whereas the real-life context facilitates client-centred rehabilitation, more collaboration and communication, and a more positive therapeutic alliance. Discussion: Findings indicate home-based rehabilitation is at least effective as a day hospital-based program. Qualitative findings indicate a more positive rehabilitation experience at home. Research examining the cost effectiveness of home-based rehabilitation is needed.
\end{abstract}

\section{Transition Success: Investigating Outcomes in the Transition From Hospital to Home Following Brain Injury}

\author{
Emily Nalder, ${ }^{1,2}$ Jennifer Fleming, ${ }^{1,2}$ Petrea Cornwell, ${ }^{1,2}$ Michele Foster, ${ }^{3}$ \\ Tamara Ownsworth ${ }^{4}$ and Melissa Kendall ${ }^{2}$ \\ 1 The University of Queensland, School of Health and Rehabilitation Sciences, Australia \\ 2 Princess Alexandra Hospital, Brisbane, Australia \\ ${ }^{3}$ The University of Queensland, School of Social Work and Human Services, Australia \\ ${ }^{4}$ Griffith University, School of Psychology, Australia
}

Tntroduction: The transition from hospital to home represents a key period $\boldsymbol{l}$ in the rehabilitation continuum for individuals with brain injury. This bridging phase between hospital and long term community integration is often an emotional one characterised by adjustment to changes in life roles and abilities. This paper aims to: a) Describe how individuals with a TBI perceive the success of the transition from hospital to home and, b) Discuss transition experiences, personal and environmental factors that are associated with the perception of transition success. Methods: A prospective longitudinal investigation was conducted with a sample of 102 individuals with 
a TBI. Data were collected using a variety of outcome measures examining psychosocial integration, sentinel events, emotional wellbeing and transition success. Measures were administered at four time points and the results analysed using multiple regression and mixed effect modelling. Results: Self-ratings of greater transition success at 6-months post-discharge were significantly associated with a higher level of psychosocial integration at 1month post discharge, while lower ratings of transition success were significantly related to increased time taken to become independent in community access and moving to a more restricted living situation. Conclusion: Along with overall levels of psychosocial integration, two key sentinel events (moving to a restricted living situation and independently accessing the community) were found to impact upon the perception of transition success. These findings highlight the need to further investigate the personal significance of these key events during the transition from hospital to home.

\title{
Do Training Strategies Influence Functional Outcome: A Sytematic Review
}

\author{
Kathryn S Hayward, ${ }^{1}$ Ruth N Barker ${ }^{2}$ and Sandra G Brauer ${ }^{1}$ \\ ${ }^{1}$ Division of Physiotherapy, School of Health and Rehabilitation Sciences, \\ The University of Queensland, Australia \\ 2 Discipline of Physiotherapy, School of Public Health, Tropical Medicine and Rehabilitation Sciences, \\ James Cook University, Australia
}

\begin{abstract}
A lmost all stroke survivors are functionally disabled after stroke. To promote functional recovery, stroke survivors are encouraged to undertake task-oriented training. To accelerate skill acquisition, other training strategies are applied during task-oriented training, such as mental practice and visual feedback. This presentation will provide the results of a systematic review with meta-analysis of training strategies, to determine the effect of these strategies on functional outcome for stroke survivors. All relevant databases were searched using combinations and variations of the terms stroke and functional recovery, along with training strategy specific terms; e.g., feedback. Randomised controlled trials that investigated the effect of a training strategy on the functional outcome of adult stroke survivors were included. A total of 33 articles were included. Mental practice had a moderate, statistically significant effect on functional outcome. Intensity of practice and feedback had a small, statistically significant effect on functional outcome. There was no evidence that any other training strategies had an effect on functional outcome for stroke survivors; e.g., bilateral movement therapy, demonstration. Therefore, further research is required to: (1) investigate training strategies which have not been rigorously investigated in an RCT design; (2) determine the critical amount of each training strategy required to produce a clinically meaningful effect on functional outcome for stroke survivors; and (3) evaluate how to best implement these strategies with a variety of task-oriented training interventions.
\end{abstract}

\section{Drivers' Ability to Anticipate Traffic Hazards After a Recent Mild Traumatic Brain Injury}

Megan H. W. Preece, Mark S. Horswill and Gina M. Geffen

School of Psychology, The University of Queensland, Australia

Tntroduction: The question of whether individuals with a recent mild trau1 matic brain injury (MTBI) are safe to drive has not been investigated, despite impaired cognition being common soon after MTBI. The aim of this 
study was to examine the effect of a recent MTBI on drivers' ability to anticipate traffic hazards. Slower anticipation of traffic hazards has been associated with higher crash rates, but this driving skill has never been assessed after MTBI. Methods: A convenience sample of 42 patients with MTBI and 43 patients with minor orthopedic injuries was recruited from a hospital's emergency department within 24 hours of injury. Participants completed a computerised hazard perception test, in which they watched videos of genuine traffic scenes filmed from the driver's point of view. They were required to use the computer mouse to click on potential traffic hazards (defined as situations were a traffic conflict between the camera car and another road user would eventually occur) as early as possible. Results: Participants with MTBI were significantly slower to identify traffic hazards than the participants with minor orthopedic injuries $(p=.03, d=.48)$. Discussion: This study provides the first indication that, within the acute stage post-injury, MTBI can be associated with impairment in a crash-related component of driving. Though further research needs to be done to clarify this finding, it suggests that individuals who present to hospital with MTBI should be advised to refrain from driving for at least the first 24 hours after injury.

\title{
CONCURRENT SESSION 5: PAEDIATRIC OUTCOMES AND INTERVENTIONS
}

\section{Developing a Metric to Measure Care and Support Needs in Children} and Adolescents With Acquired Brain Injury

\author{
Cheryl Soo, ${ }^{1,2}$ Robyn Tate, ${ }^{2}$ Vicki Anderson ${ }^{1}$ and Mary-Clare Waugh ${ }^{3}$ \\ ${ }^{1}$ Australian Centre for Child Neuropsychology Studies, Murdoch Children's Research Institute, \\ Royal Children's Hospital, Melbourne, Australia \\ 2 Rehabilitation Studies Unit, University of Sydney, Australia \\ ${ }^{3}$ Rehabilitation Department, Children's Hospital at Westmead, Australia
}

\begin{abstract}
Tntroduction: The Paediatric Care and Needs Scale (PCANS) was developed to address the lack of scales currently available for measuring support needs of children with acquired brain injury (ABI). The scale assesses supervision and physical assistance across 14 domains of everyday activities from support for personal hygiene to participation in leisure activities. This study aims to examine PCANS scores according to age and sex in a normative sample of Australian children. Methods: Participants were parents/caregivers of typically developing children aged 5-14 years $(N=300)$ recruited from a diverse range of schools in metropolitan Melbourne. Children with ABI, diagnosis of a neurological or developmental disorder, or significant medical condition were excluded. Thirty parents of children in each of the 10 age-bands, with approximately equal sex ratio were recruited. Results: Results showed a trend for PCANS support needs scores for the Home Activities domain to be the most stable across the ten age-bands. PCANS domains that showed greatest decrease in support needs with increasing age were supervision for Interpersonal Relationships and School Functioning. Compared to girls, higher total supervision support scores were found for boys aged $8-11$ years $(z=-2.1 ; p<.05)$ with no significant sex differences in younger (5-7 years) and older (12-14 years) groups. For total physical assistance scores, no significant sex differences were found across all age groups. Discussion: This study provided normative data for the PCANS using a sample of children stratified by age and sex. Findings will provide an important point of reference to guide clinical interpretation of the PCANS for assessing support needs of children with ABI.
\end{abstract}




\title{
Functional Outcome of Children with Traumatic Brain Injuries
}

Katherine Langdon, ${ }^{1}$ Anne-Sylvie Ramelet, ${ }^{2}$ Joy Knowles, ${ }^{1}$ Judith Finn, ${ }^{3}$

Simon Erickson, ${ }^{1}$ Alan Duncan, ${ }^{1}$ Gary Geelhoed ${ }^{1}$ and Jane Valentine ${ }^{1}$

1 Princess Margaret Hospital for Children, Perth, Australia

2 Hecv Sante, Hes-So University of Applied Sciences, Western Switzerland

${ }^{3}$ School of Population Health, University of Western Australia, Australia

Traumatic brain injury (TBI) is a leading cause of death and disability in 1 children. It has been contested that demographic factors and injury severity play a part in determining the functional outcomes of TBI. Aim: To compare premorbid educational status and severity of TBI with educational and functional outcomes immediately post injury and at 2 and 5 years. Method: Children with TBI aged between 0-16 years from 2000-2006 were identified by data linkage of Hospital Morbidity data, PICU database and the Acquired Brain Injury database of the Department of Paediatric rehabilitation, PMH. Injury severity was assessed by GCS and length of PTA, and functional status by WeeFIM. Educational status was assessed by educational liaison interview and level of educational support. Results: 233 children were admitted to ICU with skull fractures and, or intracranial injuries of which 23 children died and 61 children were admitted to the paediatric ward for rehabilitation. Of the children admitted for rehabilitation, mean age was 8 and the mean GCS on admission was 7. GCS was $\leq 8$ for $71 \%, 9-12$ for $26 \%$ and $13-15$ for $3 \%$ of children transferred for rehabilitation. $35 \%$ of school-aged children admitted for rehabilitation had pre-morbid cognitive, learning, or attentional problems, which increased to $56 \%$ in the months immediately following discharge. Most children required significant educational assistance to return to school. Discussion: Premorbid status and injury severity influences educational and functional outcome following TBI in children.

\section{Are Children Who Experience Traumatic Brain Injury More Likely to Engage in Criminal Behaviour During Their Adult Lives?}

\author{
Audrey McKinlay, ${ }^{1}$ L. John Horwood ${ }^{2}$ and David M. Fergusson ${ }^{2}$ \\ ${ }^{1}$ Department of Psychology, University of Canterbury, New Zealand \\ ${ }^{2}$ Christchurch Health and Development Study, University of Otago, New Zealand
}

$\boldsymbol{B}_{\text {ackground: Previously we reported an association between early mild }}$ including attention deficit and conduct disorders and problems with substance abuse over ages 14-16. Here we investigate whether TBI is associated with greater participation in criminal activities during adulthood. Method: The data reported here were part of the Christchurch Health and Development Study, a longitudinal epidemiological study of a birth cohort of 1265 children born in Christchurch (New Zealand) urban region in mid1977. Individuals who experienced a TBI over $0-21$ years $(N=266)$ were divided according to injury severity, those who had been hospitalised (Inpatient) and those who had not (Outpatient). The remainder of the cohort formed the no-injury control group. Results: Outcomes were assessed over ages 21-25 using self-reported arrests, violent offences and property offences, both before and after the adjustment for gender, SES, parental reports of behavioural problems (over age 1-5 years), combined parentteacher report of the child's conduct-disordered behaviour (7-13 years). Adjusted rates indicated that compared to non-injured individuals, both TBI groups were more likely to be arrested (Incidence Rate Ratio $($ IRR) $=2.03$ 
and $I R R=1.68)$, involved in property offences $(I R R=2.08$ and $R R=1.54)$ and violent offences (IRR $=1.35$ and IRR $=2.29)$ (all $p<.01)$. Translating into increased rates of re-offending of $35 \%$ to over $129 \%$ compared to the no-injury group. Conclusions: Over $90 \%$ of the TBI events were in the mild range. We controlled for a wide range of factors and there was still clear evidence of increased criminal activity for individuals who had experienced a TBI when compared to their non-injured counterparts.

\title{
Prediction of Cognitive Outcome 3 and 6 Months After TBI in Childhood
}

\author{
Rian Dob, ${ }^{1}$ Justin Kenardy, ${ }^{2}$ Gina Geffen ${ }^{1}$ and Vicki Anderson ${ }^{3,4}$ \\ ' School of Psychology, University of Queensland, Australia \\ ${ }^{2}$ Centre of National Research on Disability and Rehabilitation Medicine and School of Psychology, \\ University of Queensland, Australia \\ ${ }^{3}$ Royal Children's Hospital, Melbourne, Australia \\ ${ }^{4}$ University of Melbourne, Australia
}

\begin{abstract}
Tntroduction: The Glasgow Coma Score partially predicts early cognitive outcome in children who sustain a traumatic brain injury (TBI), but much unexplained variance remains. The aim of this study was to identify other predictors of cognitive outcome during the first 6 months after TBI. Based on current literature, it was hypothesised that measures of premorbid behaviour and adaptive functioning would contribute significantly to cognitive outcomes. The impact of emotional adjustment was also explored. Potential benefits of more accurate prediction of cognitive outcome include the possibility of allocating rehabilitation resources to where they are most needed and/or most likely to improve outcome. Method: The sample consisted of 70 children with TBI. Parents completed ratings of premorbid functioning within 2 months after TBI. Children underwent assessment with neuropsychological and psychological measures at 3 and 6 months postinjury. Psychometric measures were combined to yield a cognitive composite score for use as the outcome variable in analyses. Results: Analyses showed that TBI severity, as measured by GCS, accounted for $25 \%$ of variance in cognitive outcome. Premorbid and emotional adjustment variables did not account for significant additional variance in predicting outcome. Discussion: The results suggested that premorbid functioning and emotional adjustment do not impact on cognitive outcome at 3 and 6 months after TBI in childhood. These results are consistent with other studies early after TBI in childhood. Longer-term follow-up is required to fully explore the impact of premorbid behaviour and adaptive functioning, and emotional adjustment factors, on cognitive outcome after TBI.
\end{abstract}

\section{Chatroom Use of Teenagers With Traumatic Brain Injury: Presenting Results From a Systematic Review}

\footnotetext{
Andrea M. Kilov, ${ }^{1}$ Leanne Togher, ${ }^{1}$ Emma Power ${ }^{1}$ and Lyn Turkstra ${ }^{2}$

${ }^{1}$ Disability and Community Research Group, Faculty of Health Sciences, University of Sydney, Australia

${ }^{2}$ Department of Communicative Disorders, University of Wisconsin, United States of America
}

Tntroduction: There is research emerging in the areas of computer, email and Internet use amongst people with traumatic brain injury (TBI), who report a strong desire to use these tools to reduce boredom and social isolation (Todis, Sohlberg, Ficus, \& Hood, 2005; Vaccaro, Hart, Whyte, \& Buchhofer, 2007). However, little is known about chatroom use of people, and in particular, teenagers with TBI. Therefore, the aims of this systematic 
review are to: (1) investigate if there are sources that describe chatroom use of teenagers with TBI and (2) identify if chatroom training packages exist for these teenagers or their carers as chatrooms may hold great potential for improving communication and social connectivity of this population. Methods: Online database searches were performed to June 2009. A separate search was conducted on speechBITE ${ }^{\mathrm{TM}}$ (www.speechbite.com), using specific search fields. Sources were included if they contained target search terms and were relevant to the topic of chatroom use of teenagers with/out TBI. Results: 46 sources were included in the systematic review, with only 6 including the teenagers with TBI, 20 addressing chatroom use, and 18 addressing chatroom discourse. 10 sources were relevant to Internet, email, or chatroom training of people with TBI. Discussion: There is existing literature that describes social and educational benefits of chatroom use of typically developing teenagers. There is no research that describes chatroom interactions of teenagers with TBI and similarly, no chatroom training packages exist for this population. Further research in this area is warranted.

\title{
Relevance and Identity Versus Competence: The Competing Demands of Adolescent TBI Rehabilitation.
}

\author{
Lucie Shanahan, ${ }^{1}$ Lindy McAllister ${ }^{2}$ and Michael Curtin ${ }^{3}$ \\ ' School of Community Health, Charles Sturt University; The Kids' Team, South West Brain Injury \\ Rehabilitation Service, Albury, Australia \\ 2 School of Medicine, University of Queensland, Australia \\ ${ }^{3}$ School of Community Health, Charles Sturt University, Australia
}

The likelihood that paediatric traumatic brain injury (TBI) can cause persisting disability across a range of domains, and that difficulties can worsen or emerge as a child moves through to adulthood is well documented. These factors support the need for long term, specialised rehabilitation that can support adolescents and their families at developmental transitions. However, clinicians working with this population face several competing demands: (a) engaging long term rehabilitation clients during a phase of intensive executive system development; (b) responding to the needs and goals of the adolescent as well as their parents and at times, their teachers; and (c) providing a service that is considerate of the tasks of normal adolescent development. This paper presents key findings from a mixed methods study that explored the level of competence two adolescents with TBI reported in their executive functioning skills compared with the perceptions of their parents and a key teacher. Data was collected via normative assessment and clinical observation of the adolescents, and semistructured interviews with all participants. The findings highlighted the different emphasis participants placed on skill competence and confidence. Adolescents were unable to differentiate skill confidence and competence but they reported the need to feel that tasks contributed to the development of their role and identity. Parents and teachers needed to see incremental development in the adolescent's executive functioning skills to ultimately report a sense of confidence. The findings have implications for rehabilitation programming for long term paediatric clients and for service delivery approaches used with adolescent clients. 


\title{
CONCURRENT SESSION 6: EMOTION AND BEHAVIOUR
}

\section{Recognising Emotions in Music in Early Frontotemporal Dementia: Preliminary Findings}

\author{
Sharpley Hsieh, Olivier Piguet and John R. Hodges \\ Prince of Wales Medical Research Institute; University of New South Wales, Australia
}

\begin{abstract}
Tntroduction: Frontotemporal dementia (FTD) is a progressive neurodegenerative brain disease characterised clinically by abnormalities in behaviour, cognition and language. Two subgroups, behavioural-variant FTD (bvFTD) and semantic dementia (SD), also show impaired emotion recognition particularly for negative emotions. This deficit has been demonstrated using visual stimuli such as facial expressions. Whether recognition of emotions conveyed through other modalities, for example music, is also impaired has not been investigated. Methods: Patients with bvFTD, SD and Alzheimer's disease (AD), as well as healthy age-matched controls, labeled tunes according to the emotion conveyed (happy, sad, peaceful or scary). In addition, each tune was also rated along two orthogonal emotional dimensions: valence (pleasant/unpleasant) and arousal (stimulating/relaxing). Participants also undertook a facial emotion recognition test and other cognitive tests. Integrity of basic music detection (tone, tempo) was also examined. Results: Patient groups were matched for disease severity. Overall, patients did not differ from controls with regard to basic music processing or for the recognition of facial expressions. Ratings of valence and arousal were similar across groups. In contrast, SD patients were selectively impaired at recognising music conveying negative emotions (sad and scary). Patients with bvFTD did not differ from controls. Conclusion: Recognition of emotions in music appears to be selectively affected in some FTD subgroups more than others, a disturbance of emotion detection which appears to be modality specific. This finding suggests dissociation in the neural networks necessary for the processing of emotions depending on modality.
\end{abstract}

\section{The Need for a Transdiagnostic Conceptualisation of Depression and Anxiety Symptomology Following Traumatic Brain Injury?}

Cassandra Gordon and Tamara Ownsworth

Applied Cognitive Neuroscience Research Unit, Griffith Institute for Health and Medical Research, and The School of Psychology, Griffith University, Australia

Gor over a decade now researchers and clinical psychologists have been

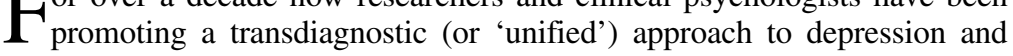
anxiety within the general population. Advocates of the transdiagnostic approach suggest that there are common underlying factors across anxiety and depression, and that these disorders may be part of the same fundamental emotional syndrome. This viewpoint may be highly relevant to the traumatic brain injured (TBI) population, as psychological distress post-TBI often presents as a range (or syndrome) of neurological-emotional reactions, rarely fitting neatly into a diagnostic category. Therefore, a transdiagnostic approach may offer an enhanced understanding of the factors which underlie depression and anxiety following TBI. This review aimed to examine existing empirical support concerning the rationale for a transdiagnostic conceptualisation of depression and anxiety post-TBI. A broad review of the TBI literature was conducted that encompassed studies investigating factors related to emotional adjustment and treatment studies targeting emotional 
adjustment. Three main lines of preliminary support for a transdiagnostic approach were identified, including; (1) high comorbidity between depression and anxiety symptoms, (2) observed treatment response for non-targeted aspects of emotional function, and (3) some common underlying neuro-cognitive, psychological and social factors found to be related to both depression and anxiety post-TBI. However, despite this preliminary support the review highlighted that research is yet to adopt a transdiagnostic approach, and specifically examine common factors contributing to depression and anxiety following TBI. Such research has the potential to guide the development of more unified and efficacious psychotherapeutic interventions.

\title{
The Effects of Posttraumatic Stress Symptoms (PTSS) on the Physical and Psychosocial Wellbeing of Adults Diagnosed With a Meningioma (Brain Tumour)
}

\author{
Maria Kangas, ${ }^{1}$ Janet Williams ${ }^{2}$ and Robert I. Smee ${ }^{2}$ \\ ${ }^{1}$ Centre for Emotional Health, Department of Psychology, Macquarie University, Australia \\ 2 Department of Radiation Oncology, Prince of Wales Hospital, Sydney, AUstralia.
}

\begin{abstract}
Tntroduction: A meningioma is one type of brain tumour that commonly occurs in adults. Individuals may experience a threat to their physical integrity due to locality of tumour, side-effects, as well as having to contend with uncertainty given the risk of tumour progression and potential threat to life. Hence, being diagnosed with a meningioma may be a stressful/traumatic life experience. There is a paucity of research which has investigated posttraumatic stress symptoms (PTSS) in this population and the effects this has on quality of life. Accordingly, one of the aims of this study was to address this issue. Method: Utilising a cross-sectional design, participants were administered a semi-structured interview and standardised questionnaires that assessed tumor-related PTSS, mood, quality of life, and social support. The Controlled Oral Word Association Test (COWAT) was also administered. Results: 69 participants with a meningioma were assessed on average 4.5 years following initial diagnosis. $16 \%$ of the sample reported clinically elevated PTSS in relation to their meningioma experience. Elevated PTSS was significantly associated with gender (males); lower COWAT scores; reduced physical and functional wellbeing; elevated tumour symptoms, fatigue and confusion; and reduced mood. The majority of individuals with elevated PTSS had tumours situated in the left hemisphere $(p=.068)$. Discussion: Findings indicate one in seven adults with a meningioma are at risk of developing chronic psychosocial and cognitive disturbances. The outcomes will be discussed in the context of developing and implementing appropriate assessment and rehabilitation services to address the unique needs of brain tumour populations.
\end{abstract}

\section{Lessons From Autobiographical Accounts of Coping Following Traumatic Brain Injury}

Melissa Kendall, ${ }^{1,2,3}$ Elizabeth Kendall ${ }^{1}$ and Heidi Muenchberger ${ }^{1}$

${ }^{1}$ Griffith Abilities Research Program, Research Centre for Clinical and Community Practice Innovation, Griffith University, Australia

2 Acquired Brain Injury Outreach Service, Queensland Health, Australia

3 Transitional Rehabilitation Program, Queensland Health, Australia

Tntroduction: Traumatic brain injury has been associated with significant difficulties in the area of psychosocial adjustment and coping. Studies of the relationship between coping and adjustment have been limited with inconclusive results emerging from discrepancies between quantitative and 
qualitative methods of study. Autobiographical accounts of the TBI experience provide rich descriptions of this relationship. Methods: A consecutive series of 52 autobiographical accounts written by people with TBI and published in the Journal of Cognitive Rehabilitation between 1986 and 1997 were examined. Based on their accounts, authors appeared to have acquired a reasonable level of adjustment with evidenced by an acceptance of their current situation. Using content analysis, the narratives were systematically searched for cognitive constructs such as beliefs, attitudes and coping responses. Results: Over 44 different coping strategies were identified in the narratives. These strategies clustered into 9 major coping styles, namely personal discovery, goal-directed, control/independence, purpose-focused, social, spiritual, day-by-day, hard work and discipline and emotional. There was strong use of coping styles that depended on the individual's freedom to explore their new identity and grieve the loss of their old identity using a trial and error process. Discussion: People with TBI wrote about a wide variety of coping strategies. Findings suggest people with TBI often cope by reconceptualising their lives using different frames of reference in exploratory, personal and experiential ways. The implications for rehabilitation professionals lie in their capacity to facilitate rather than hinder the natural course of coping that allows people to explore their new identity.

\title{
The Overt Behaviour Scale - Self Report (OBS-SR): Development of a Self-Report Measure of Challenging Behaviours Following ABI
}

\author{
and Peter Kremer ${ }^{3}$ \\ ${ }^{1}$ Diverge Consulting, Victoria, Australia \\ 2 Sydney South West Area Health Service, Australia \\ ${ }^{3}$ Deakin University, Australia
}

Glenn Kelly, ${ }^{1}$ Suzanne Brown, ${ }^{1}$ Grahame Simpson, ${ }^{2}$ Samantha Burns ${ }^{1}$

Thtroduction: This study aimed to develop and validate a self-report
behaviour-measurement tool suitable for individuals with acquired brain
injury (ABI), and to assess whether individuals' assessment of their own
behaviour matched the assessment of informants. Method: Thirty-five adults
with ABI and 35 informants participated in the study. Measures included the
Overt Behaviour Scale (OBS) for rating overt challenging behaviour, the
Mayo Portland Adaptability Inventory (MPAI) to measure adaptive skills,
and the Awareness Questionnaire to measure insight. In addition to the orig-
inal scales, a version reworded for self-completion was included. As part of
a broader evaluation of the psychometric properties of the OBS-SR, all par-
ticipants with ABI completed the self-report versions of the scales, and
informants (family member or service provider) completed the original ver-
sions of the scales in which they rated the person with ABI. Scales were
administered in a semi-structured interview format. Results: Comparison of
ratings of informants and individuals with ABI indicated that the latter
described themselves as having fewer challenging behaviours and as being
less disabled. Discrepancies between self- and informant reports were
greater in cases where insight was more severely impaired or disability was
more severe. Discussion: Self-reports of behaviour must be taken with cau-
tion as they often vary from the views of informants. Nevertheless, the
majority of individuals engaged well with the self-report process. A self-
report tool provides a foundation for discussion of problematic behaviours 
with individuals with $\mathrm{ABI}$, and for addressing discrepancies between them and informants in the context of treatment planning.

\title{
What 'Keys' Open the 'Acquired Brain Injury Cage’?
}

\author{
Christine Durham, Paul Ramcharan and Josephine Lang \\ RMIT University, Australia
}

\begin{abstract}
A career educator, then person with ABI, author of 'Doing Up Buttons', PhD student Durham examines Freeman's (1998) assertion that long term recovery from brain injury is a process of re-learning and education. 'Keys to the ABI Cage', an innovative, empowering methodology, similar to motivational interviewing (Rollnick \& Miller, 1995) encouraged participants to explore their own lived experience, raising their self-awareness and insight into $\mathrm{ABI}$ in a positive manner. Methods: Thirty people with ABI, 5 carers and 5 professionals, talked about a series of 'talk-about-cards' attached to a bird-cage, a metaphor to explore their experiences of ABI, to reflect and identify 'keys' that helped open their 'ABI Cage' - a methodological tool to examine commonality and diversity of $\mathrm{ABI}$ experience. Results: 'Keys to the ABI Cage' broke through barriers of power, shame, memory and communication difficulties. Responses included: '.. allowed me to open up my own eyes to ABI', 'What a relief!', '.. light for the dark night of the soul'. Recurrent themes included struggles with the 'old' and 'new' self, anomie, the centrality of hope, trust and the vital nature of empathetic treatment by health professionals. Discussion: 'The ABI Cage' has demonstrated significant practice as well as research relevance, showing the individuals views of themselves can be strengthened when they are given the opportunity to tell their story, reflect on their perceptions and understandings in terms of their validity (Cochran, 1987).
\end{abstract}

\section{CONCURRENT SESSION 7: SOCIETAL AND SYSTEMIC ISSUES}

\section{Which Factors Determine Who is Referred for Community Rehabilitation Following TBI?}

\author{
Gill Ta'eed, ${ }^{1}$ Clive E Skilbeck ${ }^{1,2}$ and Mark Slatyer ${ }^{2}$ \\ ${ }^{1}$ School of Psychology, University of Tasmania, Australia \\ 2 Neurotrauma Register Royal Hobart Hospital, Tasmania, Australia
}

Tntroduction: Although studies indicate that rehabilitation following trau1 matic brain injury (TBI) improves psychosocial functioning and decreases emotional distress, few patients are referred for community rehabilitation. Method: The Tasmanian Neurotrauma Register (NTR) is a population study of 1228 individuals presenting with TBI at the Royal Hobart Hospital's Emergency Department. The Community Rehabilitation Unit (CRU) is the single point of referral for adults who require access to public outpatient rehabilitation services in Tasmania. Over a 3-year period, following TBI only 54 individuals were referred by hospital or community health services to CRU, with 121 being referred by NTR research assistants. A further 248 individuals accessed private rehabilitation. These three groups were compared on a range of variables to determine the factors that predict referral. Results: Compared with the NTR-referred group, those referred to CRU by hospital or community services and those who received private rehabilitation had more severe TBIs $(p<.001)$, and were more likely to have been hospi- 
talised $(p<.001)$ following their injury. The NTR-referred group reported significantly more post-concussion symptoms $(p<.001)$ and more symptoms of anxiety and depression $(p<.001)$. NTR-referral was more likely $(p$ $<.001$ ) following assault, compared with the other groups. Discussion: In this study hospitalisation, insurance status and physical disability were more important factors in determining who was routinely referred for rehabilitation following TBI than measures of post-concussion symptoms or psychological distress. Further research is needed to determine the extent of engagement of the three groups with rehabilitation services and how this relates to measures of outcome.

\title{
Navigating the Gap Between Compensation Fund Expiry and the Provision of Ongoing Support for Adults With Traumatic Brain Injury
}

\author{
Kathy Buckler ${ }^{1,2}$ \\ ${ }^{1}$ The University of Queensland, Australia \\ 2 Gitana Consulting And Training Services, Ipswich, Australia
}

\begin{abstract}
Concern about addressing the methods for navigating a new life path beyond the acquisition of a traumatic brain injury (TBI) requires considerable input on a number of levels, not least of which includes access to funding to provide ongoing support. Depending on where they live, some Australians may be able to access no-fault funding arrangements for support, while the only recourse for others may be through fault-based compensation schemes. While there is widespread community perception that compensation funds will last for a lifetime, evidence indicates that these funds are rarely sustainable beyond 20 years post-settlement. Moreover, while the life spans of individuals may exceed what has been previously accepted, very limited research is available regarding the ways that ongoing support will be provided to adults with TBI who continue to have complex, ongoing support requirements when their compensation funds have expired. This paper draws on a current $\mathrm{PhD}$ study aimed at examining the impact of compensation fund expiry on the provision of long-term support for adults with TBI. Based on the findings from focus groups and interviews conducted with policy makers, managers of government-funded service delivery agencies, adults with TBI, and family care providers, the experience of negotiating with various agencies to provide ongoing support, both in the transition phase prior to compensation fund expiry as well as after the funds have expired, is examined. The accepted practice of crisis management, reduced service delivery and limited long-term support options provides a range of direct impacts on adults with TBI and their family care providers as they make adjustments to life beyond compensation funds.
\end{abstract}

\section{CTP Insurance Scheme Design: A Driver of Brain Injury Service System Development?}

\author{
Rosamund Harrington, ${ }^{1,2}$ Michele Foster ${ }^{1}$ and Jennifer Fleming ${ }^{3,4}$ \\ 1 Social Policy Unit, School of Social Work and Human Services, The University of Queensland, Australia \\ 2 Jacana Acquired Brain Injury Service, Queensland, Australia \\ ${ }^{3}$ School of Health and Rehabilitation Sciences, The University of Queensland, Australia \\ 4 Princess Alexandra Hospital, Brisbane, Australia
}

wo thirds of all severe traumatic brain injuries (sTBI) sustained within
Australia are attributable to road traffic accidents (RTA). Funding
received through Compulsory Third Party (CTP) Motor Accident Insurance 
Schemes can have a major influence on the choices, pathways and outcomes of people with sTBI and their families. There are, however, distinct differences in the legislative frameworks under which CTP Schemes operate within each Australian state and territory. These differences have a significant impact on service system design, and the accessibility of rehabilitation and ongoing care services for people with RTA acquired STBI and their families post injury. Despite this, there has been no published research to date comparing outcomes for people with RTA acquired sTBI across CTP jurisdictions within Australia or Internationally. This paper will examine the relationship between existing CTP Insurance Schemes and the TBI service systems which have evolved within the states of Queensland, NSW, and Victoria. Data from state-based motor accident insurance and civil liability legislation, CTP Insurance Scheme annual reports, brain injury service reviews, and published research was examined. Seven key variations in insurance scheme design, which are indicated to influence both the type of TBI service system which has evolved, and the individual pathways and outcomes for people with RTA acquired sTBI and their families in each state, will be discussed. The paper highlights the opportunities for future research in light of these findings and the potential of further research to enhance existing CTP Insurance Schemes so that they are more responsive to the needs of people affected by RTAacquired sTBI.

\title{
How the Visibility of Brain Injury Affects People's Causal Attributions and Expectations About Injured Persons
}

\author{
John McClure ${ }^{1}$, Gauranga Patel ${ }^{1}$ and Kim Wade ${ }^{2}$ \\ ${ }^{1}$ Victoria University of Wellington, New Zealand \\ 2 University of Warwick, United Kingdom
}

Tntroduction: Spurious physical markers such as scars that co-occur with brain injuries influence people's misunderstandings of the behaviours of persons with brain injury including their attributions for these behaviours. The experiment examined whether markers of brain injury shape people's causal attributions for the behaviour of a person with brain injury and their expectations that those behaviours would continue in 5 years time. Methods: Scenarios presented a photograph of an adolescent, in one condition with a head scar and in a second condition with no scar. The adolescent had suffered a brain injury and shown four behaviour changes, in sleep, anger, selfconfidence, and motivation. For each behaviour, students $(n=249)$ rated attributions to the brain injury and adolescence, and estimated the likelihood that the behaviours would still present in 5 years. Results: With no scar, participants attributed the behaviours equally to adolescence and the brain injury, whereas with the scar they attributed the behaviours more to brain injury than adolescence. Attributions of the behaviour to brain injury were correlated with the expectancy that the behaviours would persist in five years. Discussion: Although visible markers of injury such as scars are spurious indicators of severity, they shape and attributions for actions of persons with brain injury and their expectations that the behaviours will persist. These results inform strategies for correcting misunderstandings about persons with brain injury. 


\title{
Benchmarking Targets and Adjunct Target Data for Rehabilitation Outcomes in the Treatment of Brain Injury
}

Monique Berger, Janet Law, Frances Simmonds, Tara Stevermuer, and Jodie Tazelaar-Molinia

Australian Rehabilitation Outcomes Centre, University of Wollongong, Australia

\begin{abstract}
$\mathrm{A}$ ustralasian Rehabilitation Outcomes Centre (AROC) provides a national benchmarking system whose aim is to improve clinical outcomes of rehabilitation.Brain injury (BI) rehabilitation enables those who have experienced a brain injury to maximise their abilities and achieve optimal functioning. BI rehabilitation comprised $3.5 \%$ of rehabilitation episodes submitted to AROC in 2008. A benchmarking workshop facilitated by AROC on September 12, 2008 provided an open forum for discussion of functional outcomes pertinent to brain injury rehabilitation. A set of draft targets was discussed at the workshop, and after industry consultation the targets were finalised. One of the key points raised at the workshop was the desire to collect additional BI specific outcome items that could be used to inform current clinical practice. A BI adjunct dataset was developed post workshop and both targets and adjunct module were published in July 2009. The BI targets include goals for time since onset, Length of Stay (LOS), FIM change, and discharge destination. The targets and the achievement of providers at a national and sector level between July 2009 and July 2010 will be presented. The adjunct dataset includes PTA score and comment, an opportunity to capture chronic amnesia information, as well as reasons for delay in discharge (date ready for discharge, delays and date episode ended). At present one third of the designated BI units in Australia are collecting this data with others planning to commence collection shortly. Preliminary analysis of this data in association with the core AROC dataset will be presented. It is hoped that the adjunct data will contribute to improving the specificity of the BI target outcomes as well as the understanding of BI outcomes in Australia.
\end{abstract}

\section{Prioritising Research in Traumatic Brain Injury Rehabilitation}

Ornella Clavisi, Marisa Chau, Peter Bragge, Emma Tavender and Russell Gruen

National Trauma Research Institute, Monash University, Alfred Health, Melbourne, Australia

Tntroduction: Priority setting has been used by research organisations and funding bodies to strategically allocate research funds. The Global Evidence Mapping Initiative, on behalf of the Victorian Neurotrauma Initiative, identified priority research questions for Traumatic Brain Injury (TBI) rehabilitation. Methods: A number of methods were used to identify the scope of clinical issues relating to TBI rehabilitation: consultation with clinical experts, a mapping workshop consisting of patients and health care professionals, scoping the literature and an online survey. Using this information, research questions were developed and prioritised on the following domains: clinical importance, novelty and controversy. For each of these an evidence map was developed to identify which of the priority questions were adequately answered by existing research. Results: The four scoping methods identified 26 questions for TBI rehabilitation, of these 14 were identified as high priority. These questions included such issues as physical and medical conditions, health care delivery, mental health and behavioural issues, community integration, and cognition. The evidence maps showed there was only a small amount of high quality evidence (randomised controlled trials and 
systematic reviews) published for the high priority questions. Discussion: The questions identified as high priority reflect the heterogeneity of issues faced by TBI patients and health professionals in rehabilitation. Identifying priority areas and evidence maps can help research funders, policy-makers and research organisations to strategically target key areas lacking empirical evidence, and provide clinicians with information on the extent to which key clinical questions have been addressed by published or ongoing research.

\section{SATURDAY MAY 8}

\section{CONCURRENT SESSION 10: INTERVENTION OUTCOMES}

\section{Word Retraining in Early Semantic Dementia}

Sharon Savage, ${ }^{1}$ Eneida Mioshi, ${ }^{1}$ Kirrie Ballard, ${ }^{2}$ John Hodges ${ }^{1}$ and Olivier Piguet ${ }^{1}$

${ }^{1}$ Prince of Wales Medical Research Institute, Randwick, Australia

2 Speech Pathology, University of Sydney, Australia

Rackground: Semantic dementia (SD) is a progressive neurodegenerative $\boldsymbol{B}_{\text {brain disease characterised by a loss of vocabulary and general knowl- }}$ edge regarding the world. These expressive language deficits often impact on quality of life, causing distress and social withdrawal. The preserved episodic memory, attention, and visuospatial skills, provide a basis for language remediation in the short term; a finding supported by an emerging literature on name retrieval therapies. It remains unclear, however, over what length of time and which training approaches are most beneficial in delivering sustainable improvements. Methods: A Single Subject Experimental Design was used to investigate the effect of retraining words commonly used in daily living in two SD patients. Both patients were approximately 4 years into the disease and demonstrated severe anomia on testing. Photographs of items from the patients' homes were incorporated into Powerpoint presentations and sentence generation exercises. Patients were instructed to complete daily practice over either 3 or 6 weeks and were assessed weekly to monitor progress. Results: Marked improvements in naming ability were observed compared to baseline after one week, with continuing improvements over 4 to 6 weeks. Benefits of therapy were still observed 1 month after intervention but declined over time without additional practice. Naming of untrained items either remained unchanged or continued to decline over time. Performance on verbal fluency and conversational tasks provide some evidence for generalisation. Discussion: These data suggest that with regular practice, patients with SD can re-learn the names of common household items using simple word retraining exercises.

\section{Investigating the Therapeutic Benefits of Karaoke: A Speech Pathology and Recreation Therapy Group for TBI Clients}

Alanna Huck and Amanda Younis

Royal Rehabilitation Centre Sydney, Australia

$\mathrm{T}$ The benefits of music and singing have been investigated, demonstrating positive therapeutic applications for various communication disorders (Racette et al., 2006; Tamplin 2007). Previous research has also shown longterm improved effects on vocal range, intonation styles and mood in people with traumatic brain injury (TBI) (Baker et al., 2005). The motivating quali- 
ties of music and the similarities between singing and speech suggest that singing may play an important role in speech rehabilitation. Music and singing groups in dementia and stroke populations have also demonstrated positive benefits for cognitive recovery and mood states (Lesta \& Petocz, 2006; Sarkamo et al., 2008). Following TBI a combination of cognitive, physical and behavioural deficits may ensue ranging from speech, prosody or language impairments, to attention, memory, concentration and overall interactive difficulties. The relationship between Karaoke and a broader spectrum of rehabilitation goals in TBI remains largely unknown. A weekly Karaoke Group is conducted in an inpatient Brain Injury service by the speech pathologist and recreation therapist. The group provides an opportunity for clients to practise their individual rehabilitation goals. Data was collected looking at changes in performance based on speech outcome measures (MPT, pitch, volume, accuracy, and intelligibility), participation, attention and mood. The results of this pilot study will inform future service planning within our service and may provide inspiration to other inpatient settings.

\title{
Innovative Neurorehabilitation Through Creative Expression: A Pilot Project
}

\author{
Sally Ann Sainsbury, ${ }^{1}$ Elizabeth Mudri ${ }^{1}$ and Nicole Hein ${ }^{2}$ \\ ${ }^{1}$ Brightwater Care Group, Oats Street Facility, Western Australia \\ 2 Curtin University, Australia
}

R ees (2005) has noted that, in cases of acquired brain injury, 'Unless there Ris significant participation in carefully chosen activities, withdrawal, isolation, and dependency can become characteristic and therefore reinforce negative behaviours in an all-encompassing cycle of impulsivity, disinhibition, and stress-laden unawareness'. Seven individuals with acquired brain injury or stroke in a long-term rehabilitation facility, aged between 26 and 55 years, took part in a six week Art Therapy clinical initiative with a focus on 'perception of the self' as a tool to unlock healing potential and create meaningful change in overall therapeutic outcomes. In this pilot study, healing potential is 'unlocked' by using art as a vehicle to put perceptions of the self, both positive and negative, into a meaningful, symbolic context. Six different tactile and sensory art media were employed to explore self-portraiture in the form of mandalas, head and body sculptures, handprints and masks. The sessions were carried out weekly and were lead by a speech pathologist/art therapist, social worker and occupational therapy student. Tasks were always sensation-based, to facilitate visceral and unconscious connection of client to their artwork, and to allow sensation to override the cognitive processes necessary to make technically proficient works. Participants' engagement was measured by time engaged in the task, number of artworks produced and quality of social communication with therapists and other participants. Anecdotal evidence was collected during and after each session on participants' general mood, willingness to take an active role in other rehabilitation therapy tasks, and overall level of self-awareness.

\section{Cognitive Training: A Potential New Treatment for Acquired Brain Injury}

Nicola Gates and Michael Valenzuela

University of New South Wales, Australia

$\mathrm{R}_{\mathrm{a}}^{\mathrm{c}}$ esearch has shown that the brain retains its capacity for neuroplasticity across the life span into advanced age, and following acquired brain injury. Evidence also suggests that involvement in mentally and physically 
stimulating activities is associated with increased brain reserve and reduced risk of dementia. Harnessing the potential for neuroplasticity and increasing brain reserve provides the opportunity for non-pharmacological treatment. One form of mental activity, cognitive training (CT), has demonstrated some efficacy in a number of ABI populations including MCI. The purpose of this paper is twofold. First, to present a systematic review of the current research literature investigating $\mathrm{CT}$ in $\mathrm{ABI}$ populations, including current methodological issues. Second, to provide a clinical framework to implement CT programs, and critically review several commercial programs, in order to assist clinicians in selecting the most appropriate intervention for their setting.

\title{
Using a Handheld Computer to Compensate for Memory and Planning Difficulties After Brain Injury: Results From a Randomised Control Trial
}

\author{
Belinda Carr, ${ }^{1}$ Natasha Lannin, ${ }^{1,2}$ Jeanine Allaous, ${ }^{1}$ Bronwyn Mackenzie, ${ }^{1}$ Robyn \\ Tate, ${ }^{1,2}$ Alex Falcon, ${ }^{1}$ Julia Schmidt, ${ }^{1}$ lain Irving ${ }^{1}$ and Shannon Narayan ${ }^{1}$ \\ ${ }^{1}$ Royal Rehabilitation Centre Sydney, Ryde, Australia \\ 2 Rehabilitation Studies Unit, The University of Sydney, Australia.
}

\begin{abstract}
Tntroduction: Previous studies have demonstrated the effectiveness of pagers and diaries to compensate for everyday memory and planning problems after traumatic brain injury. Mainstream technologies aimed at improving organisation, planning and memory skills in the general population, such as handheld computers (PDAs), are thought to hold great potential for individuals who have suffered a brain injury. This randomised controlled trial evaluates whether PDAs, individualised by occupational therapists, improve independence in people with memory problems and executive deficits after traumatic brain injury. Methods: Randomised controlled, assessor-blinded trial of 42 participants with memory, planning, attention, and/or organisation difficulties. The experimental group used a PDA for 8 weeks, while the control group did not. All PDAs were individualised by an occupational therapist using existing and/or uploaded memory aids to support each participant (all had common features of alarm, diary, notebook and to-do list). Participants were assessed at baseline and 8 weeks later using Goal Attainment Scaling, the Prospective Memory Questionnaire, and the Rivermead Behavioural Memory Test. Results: At the time of writing this abstract (November 2009) the research team is completing the analysis of the finished study. Preliminary analyses suggest that participants who use an individualised PDA are successful in carrying out everyday activities (such as self care, self medication, and keeping appointments). Full results will be presented. Discussion: This study will provide the first-ever randomised controlled trial evidence to demonstrate that people with memory impairments from brain injury can use individualised hand-held computers. Results and clinical implications will be discussed.
\end{abstract}

\section{A Protocol for Measuring the Direct Clinical Benefit of Neuropsychological Assessment With Feedback to Patients with Multiple Sclerosis and Their Caregivers}

\author{
Wendy A. Longley, ${ }^{1,2}$ Robyn L. Tate ${ }^{1}$ and Rhonda F. Brown ${ }^{3}$ \\ ${ }^{1}$ Rehabilitation Studies Unit, Faculty of Medicine, University of Sydney, Australia \\ 2 MS Australia - ACT/NSW/VIC, Australia \\ ${ }^{3}$ School of Behavioural, Cognitive and Social Sciences, University of New England, Australia
}

Tn Australia, most neuropsychologists consider their primary clinical role as Iproviding diagnostic information on their patient's cognitive strengths and weaknesses with a view to helping guide the diagnostic formulation, care 
plan, goal-setting, and discharge planning decisions of the patient's care team, rather than using this information to deliver direct therapeutic interventions, as occurs elsewhere in the world. There is evidence that this neuropsychological information is highly valued by the patient's care team. Yet evidence of any direct benefit to the patient is based solely on their ratings of satisfaction, rather than on any measured improvements in clinical outcome. Indeed, the few studies that have measured clinical outcomes of neuropsychological assessment have failed to show any direct benefit to the patient. This lack of evidence is a problem for our profession, particularly in the current funding climate where neuropsychological assessment was excluded from the government's 'Better access to psychologists through the Medicare Benefits Schedule' 2006 initiative. This presentation will outline a protocol for measuring the direct putative clinical benefit of neuropsychological assessment with feedback using a randomised control trial with crossover. Thirty-six patients with MS and their caregivers are randomised to either treatment or wait list control groups. A broad range of psychological outcomes is measure before and after intervention, including subjective perception of cognitive impairment, mood, coping style, self-efficacy, memory compensation, and quality of relationship between patient and caregiver. Data collection is in progress, and preliminary findings will be presented.

CONCURRENT SESSION 11: FAMILY SUPPORT AND OUTREACH PROGRAMS The Family's Needs: Challenges, Complexity and Creativity

\author{
Helen Harrington, Kerry Stringer and Di Winkler \\ Summer Foundation/Epworth, Victoria, Australia
}

$\mathrm{R}$ esearch highlights both the injured person and family members frequently report they are socially isolated and their needs are unmet. Alarmingly recent research (Winkler et al., 2007) identified these problems are significantly higher for the most severely brain injured persons and their family. Families, with a member under 50 living in an aged care facility, consistently reported they felt isolated and had limited access to information. The majority of families reported not knowing what help or assistance was available, or how to gain this information. In turn this lead the family members to feeling socially isolated, increased their distress and disabled their ability to make choices. In response to these findings the Summer Foundation commenced a pilot program to implement a creative response to enable the access of information and support for this disempowered group. A collaborative approach with family members, and service providers was taken including a consultation phase interviewing 6 family members. This information gained built the foundations of the next phase of the provision of information sessions for families in both metropolitan and regional areas. The 8 completed sessions enabled family members to meet others in similar situations, and share their experiences. The family feedback and viewpoints have been collated and analysed for common themes. The feedback highlights this group of family members needs are commonly unmet, their isolation cannot be addressed by what service providers presume will help, and a creative approach is required to enable families to support each other at times of choice, allowing multiple methods of gaining information to suit the family's needs and changes. The paper aims to describe the complex pathway for the family members and highlights the process of addressing their needs. 


\title{
Implementing a Family to Family Link-Up Program for People With an Acquired Brain Injury and Their Families in Metropolitan and Regional Victoria
}

\author{
Franca Butera-Prinzi and Nella Charles
}

The Bouverie Centre, Victoria's Family Institute, Faculty of Health Science, La Trobe University, Australia

\begin{abstract}
Tntroduction: Despite time post brain injury, one of the most common negative secondary effects reported by families living with a member (child or adult) with an acquired brain injury (ABI) is the social isolation. Among the strategies that families have found useful in successfully coping and maintaining a healthy outlook includes obtaining adequate social support, information and attending support groups. The ABI Team at The Bouverie Centre will present the findings of the ABI — Family To Family Link Up (f2f Link Up) program, a unique community initiative that creates connections between families in supportive and community-oriented ways that acknowledges the social isolation, burden of care for all family members, and behavioural challenges that arise as a result of head injury, yet draw on families' own solutions, wisdoms and coping strengths in dealing with ABI. Methods: A co-operative inquiry action research methodology provided insights into the development and implementation of a f2f Link Up program. Qualitative and quantitative data were collected from families and facilitators on the effectiveness of the program and their experience of the Link Up sessions. Results: Results indicate positive outcomes for both families and facilitators and provides insight on the program implementation, benefits and constraints of an ABI Family to Family Link Up program. Discussion: The opportunity to meet other families in brief, time-limited contacts with a trained facilitator is a useful and needed addition to a range of resources that might assist families caring for a member with an acquired brain injury.
\end{abstract}

\section{Linking Siblings Through the 'Tree of Life'}

Nella Charles ${ }^{1}$ and Nadine Holgate ${ }^{2}$

${ }^{1}$ Acquired Brain Injury Team, The Bouverie Centre, Melbourne, Australia

2 Statewide Abi Paediatric Service, Melbourne Citymission, Thornbury, Melbourne, Australia

The Family to Family Link up Program (f2f Link up), developed by The 1 Bouverie Centre, aims to promote connections between families who have a family member with an Acquired Brain Injury (ABI). This presentation describes the experience of linking adolescents who have a brother/sister with an ABI using the 'Tree of Life' in a collaborative initiative between two agencies. The Tree of Life, is an approach based on narrative ideas, which highlights the strengths, skills and hopes of vulnerable children through the use of a structured, creative activity. It has a unique focus on bringing forward the knowledge and 'collective wisdoms' of children and adolescents and through this, creates a safe context to share difficult experiences. This presentation will describe the themes that emerged for siblings who were linked together from different families; the mutual support, compassion and hope fostered through the process; ideas generated about what helps siblings get through difficult times and the experience for parents hearing their children's wisdoms. While the significant and continuing impact of ABI was evident for these siblings, they reflected a maturity and depth of wisdom beyond their years. From these link-ups, siblings have been inspired to meet again to record and find ways to use their wisdoms to support other siblings. Quantitative feedback confirmed the benefits for sib- 
lings experienced from linking with other siblings. The documenting of sibling wisdoms that emerged from these link-ups also provide a rich resource for professionals in the field.

\title{
Preliminary Findings of an Internet-Based Information Intervention for Childhood Postconcussion Syndrome
}

\author{
Katherine Olsson, ${ }^{1,2}$, Justin Kenardy, ${ }^{1,2}$ Owen Lloyd ${ }^{3}$ and Jennifer Gwinn ${ }^{3}$ \\ ${ }^{1}$ Centre of National Research on Disability and Rehabilitation Medicine, AUSTRALIA \\ ${ }^{2}$ School of Psychology, The University of Queensland, AUSTRALIA \\ ${ }^{3}$ Queensland Paediatric Rehabilitation Service, Royal Children's Hospital, Brisbane, Australia
}

\begin{abstract}
Children who sustain a mild traumatic brain injury (mTBI) typically experience a good post injury recovery. However a proportion of children will continue to experience a cluster of cognitive and somatic symptoms known as Post Concussion Syndrome (PCS) following their mTBI. Research in adults has shown that the development of postconcussion syndrome is more related to psychosocial factors than neurological or injury-related factors. In children, family coping and psychosocial status also impact on children's post-injury neurobehavioural outcome. Several studies have shown that education based interventions are effective at reducing PCS in both children and adults. Recent research showing the effectiveness of internet delivered therapy, the increasing use of the internet by children, and that parents use the internet to gain information about their child's medical condition suggest that a PCS education intervention delivered via the internet is likely to be effective at reducing PCS symptoms in children. This presentation will discuss the rationale and methodology of an ongoing project assessing the effectiveness of an internet information provision intervention for PCS in children. Preliminary findings will be discussed.
\end{abstract}

\section{Ten Years On: Outpatient and Outreach Services for Children and Adolescents With Acquired Brain Injury in Queensland}

Margaret Rae

Queensland Paediatric Rehabilitation Service, Brisbane, Australia

The Queensland Paediatric Rehabilitation Service provides comprehensive 1 programmes for children and adolescents with acquired brain injury in Queensland. The focus of this paper will be a qualitative overview of our extensive outreach programme to 8 regional centres and the range of outpatient services available to provide flexible family focused long-term follow-up until young people transition to adult life. Regular surveys to families, stakeholders and our own staff as well as a Participation Questionnaire have added to our evidence generated by clinical practice. Key factors contributing to successful outcomes include (a) provision of multidisciplinary, goal directed, individualised assessment and intervention (b) outcome measurement and focus (c) coordination both within the service and with other medical and support agencies and (d) comprehensive liaison with key stakeholders, for example, schools and insurers. Theoretical frameworks and models of service have been developed and reviewed over the years in response to evolving and changing caseloads, feedback from consumers and a number of state and regional factors. Results of surveys to families, teachers and regional paediatric clinicians will be presented as well as feedback from families about their child's participation at home, school and community. A major challenge to the provision of 
a long-term support service is the increasing patient numbers. Workload management strategies as well as other emerging contemporary issues such as adolescent health, transition, models of service delivery and management of mild traumatic brain injury will be outlined with reference to planning for paediatric rehabilitation services in the new Queensland Children's Hospital.

\title{
To The Back of Bourke? Brain Injury Rehabilitation in Remote Areas New South Wales
}

Virginia Mitsch

GMCT Brain Injury Rehabilitation Directorate, New South Wales, Australia

Tn Australia, health outcomes have been shown to become worse as remoteIness increases (Phillips, 2009). Rural disadvantage extends beyond health to disability, with people with disabilities in rural areas experiencing significantly more social problems and access to fewer resources (Kuipers, Kendall, \& Hancock, 2001) when compared to metropolitan. In NSW rural brain injury rehabilitation programs (BIRP), developed in the early 1990's continue to provide brain injury rehabilitation and case management services in rural NSW. Rural BIRP clinicians have reported concerns and issues in meeting the rehabilitation needs of persons with an acquired brain injury who resides more remotely, that is a significant distance from a rural program. The 'Remote and Indigenous Brain Injury Rehabilitation Service Delivery' project is a joint initiative of GMCT-Brain injury Rehabilitation Directorate and the Rural Rehabilitation Research on Brain Injury (RRR$\mathrm{BI})$. The aim of the project is the development of possible models for delivery of brain injury rehabilitation in remote areas NSW via the investigation of the equity of access to services by people aged 5-65 years with a severe acquired brain injury residing in remote NSW. In addition the project seeks to explore any additional barriers experienced by Aboriginal persons to the access and use of brain injury rehabilitation services in remote areas of NSW. This paper will present the involvement of consumers and clinicians in the journey from consultation to recommendations in this solution focused project and the gaps and issues identified with potential solutions.

\section{CONCURRENT SESSION 12:}

\section{SERVICE DELIVERY AND PROFESSIONAL PRACTICE ISSUES}

\section{A Clinic for Multiple Sclerosis: Providing Evidence-Based Practice}

\author{
Rognvald (Ron) Dick \\ Waikato Hospital, Hamilton, New Zealand
}

\begin{abstract}
$\mathrm{M}$ ultiple sclerosis (MS) is a complex neurological condition that often requires a multidisciplinary approach to treatment. In an outpatient clinic setting people with MS were making multiple trips to be seen by different disciplines. In order to develop a more patient focussed assessment and treatment approach a clinic was established in 2003 to co-ordinate services by having one assessment visit where the person with MS would see the different disciplines on the same day. If treatment needs are identified the patient returns at a later date for one on one treatment intervention. The establishment of the MS Clinic resulted in a best practice award for quality and innovation. Strong links with the community based MS Society and to other hospital and community based services are maintained which provides a greater holistic approach to overall patient care and wellbeing. With regu-
\end{abstract}


lar clinic reviews the patient's MS is monitored and interventions applied when appropriate, with the aim of maintaining a person functioning independently in the community with the appropriate supports and equipment in place. Since the clinic's inception in 2003 it has undergone continual review with changes occurring to better meet the needs of those with MS. In 2005 the patients on the database were surveyed to measure the clinic against client satisfaction. As part of the continual monitoring and progression of the clinic a second review of the clinic has been carried out, with the results being compared to the first patient survey. Information on the MS Clinic will be presented along with the results of the patient survey, to provide evidence that dedicated clinics provide a value-based service to people with a neurological condition.

\title{
Evaluating Clinical Practice and Developing a New Model
}

\author{
Robyn L. Tate, ${ }^{1}$ Christine M Taylor, ${ }^{2}$ Vanessa H Aird² and Audrey Mccarry ${ }^{2}$ \\ ${ }^{1}$ Rehabilitation Studies Unit, Northern Clinical School, Sydney Clinical School, \\ University of Sydney, Australia \\ 2 Speech Pathology Service, Brain Injury Rehabilitation Unit, Royal Rehabilitation Centre Sydney, Australia
}

Tntroduction: In the area of acquired brain impairment it is usual practice for treating clinicians to evaluate a patient's functional status and use the results to plan and implement a therapy programme. It is our impression, however, that it is rare for clinicians to conduct post-treatment evaluations to determine whether treatment has been successful. It is even rarer for the therapy programme to implement control conditions so that treatment effects can be distinguished from spontaneous recovery. Methods: The present study has three aims: (1) to develop a new model of clinical practice addressing the above problems and use it to (2) audit clinical practice and (3) trial its implementation. The model incorporates the main features of the International Classification of Functioning, Disability and Health, combined with singlecase methodology. It is hierarchically based, with 7 levels representing increasingly rigorous therapeutic interventions. Results: A 12-month audit of 39 consecutive cases in a speech pathology service was conducted. Interrater agreement on level of intervention using $25 \%$ of the protocols was $90 \%$. Results confirmed initial impressions, with the majority of protocols scored at Level 3 of the model (pre-assessment with generic instruments, then therapy, but no post-therapy assessment). The model, using a multiple baseline across behaviours design (Level 7), was applied to a patient with impairments in language, targeting length of utterance. Results demonstrated improved functional language outcome that was specific to the therapy. Discussion: This model of clinical practice provides a rigorous method which the therapist can use to demonstrate whether improvement in the patient's level of function has occurred and whether that improvement can be attributed specifically to the therapy rather than spontaneous recovery. 


\title{
Translating Evidence Into Practice in Brain Injury Rehabilitation: What Evidence?
}

\author{
Annie McCluskey, ${ }^{1,2,3}$ Sally Bennett ${ }^{4}$ and Merrill Turpin ${ }^{4}$ \\ ${ }^{1}$ Community Based Health Care Research Unit, Faculty of Health Sciences, \\ The University of Sydney, Australia \\ 2 The Royal Rehabilitation Centre Sydney, Ryde, Nsw, Australia \\ 3 The National Stroke Foundation, Australia \\ ${ }^{4}$ Division of Occupational Therapy, The University of Queensland, Australia
}

\begin{abstract}
Thtroduction: Evidence-based practice requires clinicians to translate robust evidence into practice, ideally from randomised controlled trials and systematic reviews. Clinicians in brain injury rehabilitation do not have the benefit of national guidelines or multiple systematic reviews to inform practice. Furthermore, some teams provide services to multiple diagnostic groups, making service delivery particularly complex. The aim of this study is to explore how health professionals apply evidence in paediatric and adult brain injury rehabilitation. Methods: A mixed methods design has been used. Data collection methods include semi-structured interviews, a focus group and selfreport survey. First, individual interviews and a focus group were conducted with a purposive sample of 30 allied health, medical and nursing professionals, working across two services in New South Wales (NSW) and Queensland. Second, self-report surveys were completed by a convenience sample of 200 allied health professionals from NSW and Queensland, before they attended a knowledge translation workshop. Surveys relevant to brain injury rehabilitation are being analysed, and will be integrated with qualitative data. Descriptive statistics, categories and themes will be presented. Results: Data analysis will be completed by April 2010. Preliminary analysis suggests that professionals use different strategies to (a) stay up to date; (b) make decisions about changes to local protocols for screening, assessment and intervention; (c) measure outcomes; and (d) monitor service quality. Discussion: Conference delegates will learn how some professionals and teams are using evidence in to inform practice, in the absence of national guidelines.
\end{abstract}

\section{Misunderstanding Psychometrics: Impediments to More Accurate Neuropsychological Assessments and Treatment Interventions}

\author{
Stephen C. Bowden ${ }^{1,2}$ \\ ${ }^{1}$ Department of Psychology, University of Melbourne, Australia \\ ${ }^{2}$ Department of Neurological Sciences, St. Vincent's Hospital, Melbourne, Australia
}

- $f$ fective neuro-rehabilitation services depend on the rational identification

Dof patient needs and development of valid interventions. Both of these aims presuppose highly valid assessments. The practice of clinical neuropsychology enjoys diverse empirical underpinnings. Although the importance of systematic research in treatment interventions is appropriately valued, less well understood is the scientific basis of valid assessment practice known as psychometrics. Psychometric methods can be defined as the application of psychological test theory to evaluation of individual patient needs including (1) identification of treatment eligibility and (2) evaluation of treatment response. In this presentation, some common myths regarding psychometrics will be discussed. One common myth involves the idea that rigorous application of standardised tests, including standardised scoring, interferes with the clinical craft and that clinical intuition provides a superior form of validity. This myth reflects a misinterpretation of the purpose of objective assessment. Psychological tests are merely a vehicle by which clinical observations are 
turned into scientifically useful information. Other myths include the idea that psychometrics (1) requires a fixed battery approach, (2) involve blind test-data interpretation without regard to other clinical information, (3) is limited to the interpretation of IQ scores, and (4) requires interpretation of composite scores which have limited clinical validity. Uncritical acceptance of these misunderstandings may lead any neuropsychologist to neglect one of the most important classes of scientific criteria intended to improve the accuracy of clinical opinions and treatment interventions. Psychometric neuropsychology, accurately construed, should be endorsed by all scientifically-minded neuropsychologists.

\title{
Outcomes From Community-Based Rehab in New South Wales
}

Helen Badge

Greater Metropolitan Clinical Taskforce (GMCT) Brain Injury Rehabilitation Directorate, Liverpool Hospital, Sydney, Australia

Tntroduction: The GMCT BIRD initiated the Community Outcomes Project (COP) in 2006 to evaluate the 14 community brain injury rehabilitation services in NSW. Evaluating community based brain injury rehabilitation services is a significant challenge. There is no consensus regarding which outcome measures to use and a lack of evidence regarding effectiveness of brain injury rehab in community. Rehabilitation services need to provide high quality evidence base rehabilitation. Improving evidence based practice through evaluation studies and the routine use of objective outcome measures is considered best practice. The COP selected two measures to pilot as routine outcome measures for adult clients to support service evaluation. Methods: Two clinically appropriate outcome measures were selected for the pilot through a process involving clinician and consumer consultation. The measures selected included the MayoPortland Adaptability Inventory (MPAI) and Sydney Psychosocial Reintegration Scale (SPRS). The pilot project to implement and evaluate these measures involved completing repeated outcome assessments using both measures over 6 months for prospective new referrals over a 4-month period. Basic injury and demographic information was also collected. BIRP clinicians received training in the administration and interpretation of the measures. A resource manual was also developed to support the pilot. Results: Results will be presented including responsiveness to measure change, effect size, qualitative assessment of clinical utility, and results of Rasch analysis of each scale. Discussion: The relevance and clinical utility of the two measures will be discussed. Implications for providing quality brain injury rehabilitation services in NSW will also be reviewed.

\section{Measuring Goal and Outcome Achievement in Community-Based Case Management for People With an ABI Using Mayo-Portland Adaptability Inventory-4 (MPAI) and Goal Attainment Scales}

\author{
Rebekah Loukas and Rachel Merton \\ Brain Injury Association of NSW Inc., Australia
}

The Brain Injury Association of NSW commenced a case management and brokerage service in October 2008. The model is unique in NSW, as a community-based service designed to meet the complex and individual needs of people with an acquired brain injury who have not met entry crite- 
ria for existing services. Consequently, the service's clients have a range of complex needs, not just for clinical and community care, but for social contact. Many have been isolated in the community without services for years, and in some cases, decades. The service incorporates a comprehensive assessment of both clinical and social goals, which are regularly reviewed as the person's individual service plan is implemented. Fifteen Brain Injury Association of NSW clients from a pool of 24 were recruited aged from 25 to 55 years. Primary outcome measures were the Mayo-Portland Adaptability Inventory-4 (MPAI) and categories of goal attainment, with assessments pre and post six months case management intervention. Initial results indicate positive trends following intervention with the MPAI appearing most sensitive to improvement in daily function, with $t$-test analysis confirming significance. A range of issues emerged regarding approaches to achieving different kinds of personal goals through this intervention and will be reviewed. To conclude the presentation suggestions regarding the implications of case-management intervention, and recommendations for good practice in community-based case management for people with an acquired brain injury will be discussed.

\title{
POSTER PRESENTATIONS
}

\section{The Impact of Traumatic Brain Injury (TBI) on Adolescent Friendships}

\author{
Rochelle Chandler, Michael Curtin and Jane Murtagh \\ Charles Sturt University, Australia
}

The complexities of a traumatic brain injury (TBI) are known to impact on an individual's friendships. A lengthy recovery process, hidden impairments, changes in identity, and social skill impairments are common consequences of a TBI that can contribute to the loss of friendships. The consequences of a TBI on friendships are even more significant during the period of adolescence due to the emphasis on social development at this stage of the lifespan. This study explored the impact of a TBI on adolescent friendships for a single female adolescent five years post TBI. Semi-structured interviews were used to obtain data from the adolescent with a TBI, her parents, and nominated friend. Transcripts were analysed using an interpretive phenomenological approach. Analysis revealed four main themes. These were I was a different person before my accident, which details the participants loss of identity since her TBI and the impact this has had on her friendships; I'm not up to what I used to do, describes how social skill impairment and fatigue has impacted on the participants ability to engage in her friendships; I am an intruder, explores the challenges the participant faced resuming her friendships following her TBI; and Friends aren't friends, highlights the participants realisation of who her 'true friends' were following her TBI. Consistent with other research, the results of the current suggests that maintaining adolescent friendships following a brain injury is complex, and requires health professionals to implement interventions that foster friendships and prevent social isolation from occurring. 


\title{
Utilising Self-Awareness Strategies as the Underlying Framework for a Social Communication Skills Treatment Program: In-Depth Case Analyses
}

Petrea L. Cornwell, ${ }^{1,2}$ June Y.Y. Bay ${ }^{1},{ }^{1,3}$ Jennifer M. Fleming, ${ }^{1,3}$ Kerrin Watter $^{2}$ and Carly Freebairn ${ }^{2}$

1 School of Health and Rehabilitation Sciences, The University of Queensland, Australia

2 Speech Pathology Department, Princess Alexandra Hospital, Brisbane, Australia

${ }^{3}$ Occupational Therapy Department, Princess Alexandra Hospital, Brisbane, Australia

\begin{abstract}
Tntroduction: Impaired self-awareness in people who have had a traumatic brain injury (TBI) often hinders progress in, and outcomes from rehabilitation. Social communication treatment is one particular area where reduced self-awareness is thought to be a major impediment to positive outcomes. This pilot study investigated the effect of an intervention that aimed to facilitate improved social communication skills through a treatment framework based on self-awareness strategies. Methods: Program evaluation was completed using a pre- and post-treatment design. Two participants with TBI completed nine weeks of treatment with 2-hour group sessions each week, where the group consisted of participants with various acquired brain injury aetiologies. Assessments completed evaluated social communication skills and self-awareness. Results: Case analyses revealed preliminary support for the effectiveness of this program. Improvements in social communication skills and self-awareness were found for both participants. Different patterns of resolution were seen across participants. One individual demonstrated considerable change in self-awareness with only a small change in social communication abilities, while the second participant made substantial gains in social communication against a background of small changes in awareness. Discussion: These preliminary findings demonstrate that using a treatment framework based on building self-awareness in people who have had TBI simultaneously with other rehabilitation goals has promise. The differing outcomes post-treatment between the two participants will be explored in relation to their baseline social communication and self-awareness abilities. This study has practical implications for all rehabilitation clinicians in regards to the structuring of treatment programs for individuals with impaired self-awareness as one facet of their complex presentation.
\end{abstract}

\section{A Coordinated Approach to Student Clinical Education and Slow-Stream ABI Rehabilitation}

Kate Dornan and Kristylee Sharp

Brightwater, Oats St, Brain Injury Rehabilitation Service, Perth, Australia

B rightwater, Oats St, is a not-for-profit organisation providing residential and community-based rehabilitation services to people aged between 18 and 65 with an acquired brain injury. The rehabilitation program is facilitated by a multidisciplinary team comprising of allied health staff, nurses and care workers. In 2009, the OT department at Oats St entered into an agreement with Curtin University to host ongoing final year OT students. From January to December, two OT students simultaneously completed consecutive 7-week placements resulting in continuous OT student presence throughout the year. Under the guidance of supervising therapists, students were able to develop and deliver therapeutic programs, complete projects and assist therapists with research. Our presentation aims to demonstrate how the consistency and coordination of the student program facilitated the provision of therapy programs specific to clients in slow-stream acquired 
brain injury rehabilitation. Outcomes of the program were: (1) enhanced consistency in delivery of program and increased repetition of individual therapy programs essential to ABI rehabilitation; (2) increased frequency of programs able to be delivered; (3) increased number of programs able to be carried out for each client; (4) students were able to provide additional skilled support to the therapy programs otherwise carried out by untrained care workers and therapy assistants; (5) students supported a therapist to access updated, evidence-based practice to guide program development; (6) students carried out research and projects in specific areas such as sensory stimulation, insight and awareness and outcome measures for the service.

\title{
Severe Stroke - How Can We Promote Recovery: A Systematic Review
}

\author{
Kathryn S Hayward, ${ }^{1,2}$ Ruth N Barker ${ }^{1}$ and Sandra G Brauer ${ }^{2}$ \\ ${ }^{1}$ Discipline of Physiotherapy, School of Public Health, Tropical Medicine and Rehabilitation Sciences, \\ James Cook University, Australia \\ 2 Division of Physiotherapy, School of Health and Rehabilitation Sciences, \\ The University of Queensland, Australia
}

\begin{abstract}
A growing body of evidence suggests that task-oriented training promotes upper limb recovery after stroke. However typically, stroke survivors with severe paresis have been unable to participate due to a lack of underlying movement. As a result interventions have been developed that aim to enable stroke survivors with severe paresis to participate in task-oriented training. This presentation will outline the results of a systematic review that investigated the effectiveness of interventions designed to promote upper limb recovery in stroke survivors with severe paresis. All relevant databases were searched using combinations and variations of key terms related to stroke, severe paresis and task-oriented training. Randomised controlled trials which investigated the effect of an upper limb intervention on function in adult stroke survivors with severe paresis were included. Seventeen articles met the inclusion criteria and were grouped into one of three intervention categories: robotic therapy, electrical stimulation or 'other' therapy. A 'best evidence synthesis' indicated strong evidence that robotic therapy produces a large beneficial effect on upper limb function and limited evidence that electrical stimulation and 'other' interventions produce a large beneficial effect on upper limb function. There is no evidence that these interventions influence use of the arm in everyday tasks. Ultimately, the effectiveness of these interventions will be dependent on whether they lead to restoration of function to the point at which practice of everyday tasks is possible.
\end{abstract}

\section{Reflections on the Value of a Dedicated Research and Development Position in Brain Injury Rehabilitation}

\author{
Melissa Kendall1,2,3 and Ray Quinn' \\ ${ }^{1}$ Acquired Brain Injury Outreach Service, Queensland Health, Australia \\ 2 Transitional Rehabilitation Program, Queensland Health, Australia \\ ${ }^{3}$ Griffith Abilities Research Program, Research Centre for Clinical and Community Practice Innovation, \\ Griffith University, Australia
}

Tntroduction: Rehabilitation services often struggle to conceptualise and conduct applied research 'at the coalface' and then translate research findings into practice. Experienced researchers frequently engage with rehabilitation services in a transitory fashion when employed by universities or health departments in temporary roles funded by specific research grants. 
This method of engagement does little to foster research that is both servicedriven and translational. The Acquired Brain Injury Outreach Service (ABIOS) has a designated Research and Development position that was developed to redress this issue. Methods: The position was conceptualised as part of an initial 5-year service development grant in the establishment of ABIOS, marketed as an integral part of service delivery and development and subsequently funded substantively with recurrent health department funding. A task analysis was conducted to identify the ways in which the position contributed to research translation and service development. Results: The position delivers specialist research leadership, coordination and support to community rehabilitation services for people with brain injury. This involves provision of specialist clinical research knowledge, education and support to health practitioners combined with the coordination and conduct of clinical research projects entrenched within strategic research agendas aimed at enhancing client/stakeholder outcomes and building capacity in the community rehabilitation sector. Discussion: The dedicated position offers a medium through which collegiate research proliferates in service and practice driven arenas with collective researcher/clinician ownership of research conceptualisation, conduct and translation. The position has facilitated the growth of evidence-based approaches to clinical problem-solving and the implementation of quality improvement cycles.

\section{Individuals With Asperger's Impaired on Simple But Not Complex Theory of Mind Tasks}

Danielle Mathersul and Skye McDonald

School of Psychology, University of New South Wales, Australia

Thtroduction: Individuals with Asperger's are characterised by severe impairments in a broad range of psychological functions, particularly social interaction. There has been wide support for a Theory of Mind (ToM) deficit in Asperger's; however, this claim has not gone unchallenged. Importantly, there are arguments over whether the most commonly used tests of ToM are ecologically valid. The Awareness of Social Inference Test (TASIT; McDonald, Flanagan, \& Rollins, 2002) uses video vignettes depicting conversational exchanges to assess the ability to understand more subtle conversational inferences, providing closer correlates to real-life situations than traditional paper-based ToM tasks. While TASIT has been used extensively within the traumatic brain injury population and differentiates dementia populations, it has yet to be applied to Asperger's. Methods: 10 adults with Asperger's (8 males; mean age 36.7, mean FSIQ 116.7) were tested on TASIT. Results: Compared to TASIT norms, individuals with Asperger's had deficits on paradoxical sarcasm $(t=3.16, p=.03)$ and lies $(t=2.71, \mathrm{p}=.05)$. There were trends towards deficits in understanding what others were doing $(t=2.59, p=.06)$ and feeling $(t=2.59, p=.06)$, but no other deficits on the social inference sections. Discussion: Individuals with Asperger's do not have deficits in simple ToM, but rather, show deficits in understanding more complex mental states of others such as deception and paradoxical sarcasm. 


\title{
Bike Riding in Rehabilitation
}

Michael Pforr

Brightwater Caregroup, Oats Street Brain Injury Rehabilitation Service, Perth, Western Australia

\begin{abstract}
$\mathrm{A}$ bike-riding program was developed to facilitate the rehabilitation of adults between the ages of 18-65 who have sustained an acquired brain injury. Aims: to improve physical strength, endurance and flexibility; to improve balance reactions; to increase confidence with motion; to stimulate executive functions such as problem solving, planning; to increase community engagement; to provide a social activity; to enable a transport option. Funding was obtained for several three wheel bikes to work alongside two wheeled bikes. Small modifications were also made to enable trunk and ankle support. Program: Clients were assessed for their bike and staff support needs. The bike-riding session would occur on designated cycle paths with sufficient staff to client safety ratio. Staff included physiotherapists, therapy assistants, care workers and students with aim to have the program facilitated by any of these trained individuals. Clients would progress by increasing distance or by decreasing support needs. Outcomes: The bike program has continued weekly for approx two years. Physical gains have been achieved. Progression of abilities: From 3-wheel to 2-wheel, from assisted to supervised riding, from $100 \mathrm{~m}$ to $5 \mathrm{~km}$ improvements
\end{abstract}

\section{Neurobehavioural Approach To Facilitate One Man, Twenty Years' Post Brain Injury, In His Transition From Residential Care}

\author{
Anne Marie Sarandrea ${ }^{1}$ and Michael Oddy ${ }^{2}$ \\ ${ }^{1}$ ABI service, a joint venture between Royal Rehabiltation Centre, Sydney, and the Brain Injury \\ Rehabilitation Trust, United Kingdom \\ 2 Brain Injury Rehabilitation Trust, United Kingdom
}

Tn New South Wales, an initiative called Young People In Residential Aged Care facilities (YPIRAC), together with Ageing, Disabilities and Home Care (ADHC) Department of Human Services, has provided an opportunity for adults with severe Acquired Brain Injury (ABI) to move from residential care into supported accommodation. The transition out of residential care facilities requires significant assessment and rehabilitation to enable the individual with ABI to increase their independence. The neurobehavioural approach has been applied to a client who has an ABI, characterised by severe cognitive impairments, challenging behaviours, functional and physical difficulties. This paper will describe the application of the neurobehavioural approach, in the context of social rehabilitation, which included the development of individualised goals. Outcome measures were used at the beginning of assessment and at 12 weeks. The neurobehavioural approach has been integral in allowing an individual who is 20 years postinjury, to develop realistic goals for his transition into supported accommodation. He has been able to develop skills for maximising his participation and increasing his level of functioning. With assistance from Direct Support Workers (DSWs), he is now able, in his own words: 'to go on outings and be happy'. 


\section{Neurobehavioural Rehabilitation in Australia}

Anne Marie Sarandrea ${ }^{1}$ and Michael Oddy ${ }^{2}$

1 ABI service, a joint venture between Royal Rehabiltation Centre, Sydney, and the Brain Injury Rehabilitation Trust, United Kingdom

2 Brain Injury Rehabilitation Trust, United Kingdom

The ABI service opened in early 2010, and is a joint venture between the 1 Brain Injury Rehabilitation Trust (BIRT) from the UK and Royal Rehabilitation Centre Sydney (RRCS). The service provides intensive assessment and uses the neurobehavioural approach to the social rehabilitation of adults with an acquired brain injury (ABI), who may present with a range of significant impairments in cognition, behavioural and emotional regulation, and physical function. This paper will describe the neurobehavioural model of service delivery, which is led by the clinical psychologist and supported by a multi-occupational team comprised of an occupational therapist, physiotherapist, speech pathologist, nurse unit manager and direct support workers. The neurobehavioural model uses learning process that remain intact (such as procedural learning and implicit memory), behavioural methods to address behavioural and emotional regulation and rehabilitation practices such as functional goal-setting, the use of structured and closely specified routines and the use of compensatory strategies. All these are aimed at increasing levels of independence and participation. Direct support workers act as environmental facilitators. Family education and support are provided through a group program and family members are invited to participate in their loved one's neurobehavioural program during their stay in the centre. In order to maintain gains, the multi-occupational team will provide input as the individual with ABI transitions to live in supported accommodation in the community. The service is still in its early stage of development and will undergo an evaluation of its outcomes as it evolves.

\section{The Methyl-Folate Trap and Cognition in Children with Severe Cerebral Palsy: A Case in Question}

NIKEE Schoendorfer, ${ }^{1,3}$ Roslyn Boyd, ${ }^{2,3}$ LUIS Vitetta, ${ }^{3,4}$ and PSW Davies ${ }^{1,3}$

1 Children's Nutrition Research Centre, The University of Queensland, Australia

2 Queensland Cerebral Palsy and Rehabilitation Research Centre, The University of Queensland, Australia

3 The University of Queensland, School of Medicine, Australia

${ }^{4}$ Centre for Integrative Clinical and Molecular Medicine, The University of Queensland, Australia

Tntroduction: Methylation is indispensible for many processes including cognition and DNA synthesis, which are key issues in marked cerebral palsy (CP). Research in other populations has demonstrated methylation capacity related to cognitive outcomes. Methods: Blood was collected from children with marked CP $(n=25)$, either orally $(\mathrm{O})$ or enterally fed via percutaneous endoscopic gastrostomy (PEG) and healthy controls $(n=25)$. Concentrations of red cell folate (RCF), methylmalonic acid (MMA) indicating B12 deficiency and mean cell volume (MCV) were measured, as well as homocysteine (Hcy), cystathione, choline and betaine as indicators of methylation cycle function. Results: Analyses via t-tests showed Hcy increased in $\mathrm{O}($ mean $( \pm \mathrm{SD})=6.28(1.81 \mu \mathrm{mol}), p=.01)$ and PEG $(6.03$ (1.28), $p=.03)$, versus controls 5.07 (0.98). Higher MMA was found in controls (157 (54), $p<.00)$ and O (141 (101), $p=.13)$, versus PEG 88(21). RCF was raised in PEG $(1422(70 \mathrm{nmol} / \mathrm{L}), p<.00)$ versus $\mathrm{O} 843(80)$ and con- 
trols 820 (43). MCV z-scores were elevated in PEG $(3.1(1.8) p<.00)$ and O $1.1(1.1) p<.00)$ versus controls $-0.2(1.1)$. Discussion: Raised MCV is generally indicative of folate or B12 deficiency. Significantly elevated folate and MCV levels in PEG along with B12 sufficiency, may suggest folate is trapped irreversibly in the inactive form 5-methyltetrafolate and subsequently left unavailable for other folate dependent physiological functions. Well-documented malnutrition issues in orally fed children with severe CP may explain differences between CP groups. These data support the hypothesis of potential dysregulation in the methylation cycle, although more research is needed to elucidate definitive conclusions.

\title{
Open Your Eyes to ABI: Raising Awareness in Educational Facilities About the Hidden Disability of Acquired Brain Injury
}

\author{
Nadine Sexton and Kelli Van de Water \\ Monash University and Spot for Kids, Melbourne, Australia
}

\begin{abstract}
Tntroduction: Following an acquired brain injury, there may be long-term implications for cognitive, emotional and physical functioning. ABI research shows that young people may be negatively affected in their occupational roles of student, relationships and employment. Accessible and relevant support to educators is important for the achievement of positive long-term outcomes. The SPOT for Kids resource aims to support educators by providing accessible information about $\mathrm{ABI}$ and the possible implications for students. The presentation will explain the existing resource and the evaluation that was conducted. Project objectives included: Evaluating the usefulness of the SPOT for Kids booklet, increasing the agency manager's knowledge of best practice in educating educators of students with an ABI, improving the relevance and quality of the current resource, increasing the awareness of the resource within educational facilities. Methods: A survey was developed and administered to a group of educators working with students with an ABI. A number of survey participants also participated in interviews. Results: Survey results suggested that all educational facility staff should have access to the information provided by SPOT for Kids. The preferred methods of delivery were CD-ROM, DVD and website. Interview results suggested that professional development workshops were a highly desirable strategy. Transitional videos were not a strategy that was supported as they were believed to be impractical. Discussion: This study has benefits for professionals in understanding the need for improved methods of information delivery to educators simultaneously working towards improving the outcomes of students with an ABI.
\end{abstract}

\section{High-Level Language Assessment Following Traumatic Brain Injury: Methods in Clinical Practice}

Joanne Steel, Alison Ferguson and Elizabeth Spencer

School of Humanities and Social Science, University of Newcastle, Australia

\begin{abstract}
ssessment of subtle cognitive-communication impairments after TBI is A perceived to be a challenging area of speech pathology (Turkstra, Coelho, \& Ylvisaker, 2005). High-level language (HLL) deficits can be difficult to detect, describe and measure by standardised or nonstandardised testing, however left untreated they may have a significant impact on social and vocational outcomes. There is little information available about optimal
\end{abstract}


assessment procedures. The few reviews of current speech pathologists' assessment practices within TBI have been survey-based studies with a focus on the range of tests used rather than how they are utilised. The aim of the present research was to discover how HLL deficits present after TBI were identified and assessed by speech pathologists. The study used a descriptive qualitative design to explore the clinicians' perspectives of the assessment process. Participants were two speech pathologists specialising in TBI rehabilitation. Observations of assessment sessions were undertaken and subsequent in-depth interviews investigating the clinical reasoning process were recorded. Transcriptions were coded into categories using NVivo qualitative design software. Main categories were definition of HLL, clinical methods used (multiple-perspective, hypothesis testing, collaboration), and how challenges in assessment of HLL were addressed (identifying changes, differentiating from typical, client factors and limitation of tools). Assessment of HLL following TBI requires specialised clinical reasoning skills and knowledge of TBI-specific challenges. The results of the study highlight the importance of further research within this area of communication in order to inform practice for assessment and raise awareness of the complex clinical issues involved.

\title{
The Potential Unlimited Programme: A Review of Pilot Research and Future Directions
}

\author{
Matt Thomas ${ }^{1,2}$ and Lucie Shanahan ${ }^{3}$ \\ 1 School of Psychology, Charles Sturt University, Bathurst, Australia \\ ${ }^{2}$ Centre for Inland Health, Charles Sturt University, Australia \\ 3 The Kids' Team, South West Brain Injury Rehabilitation Service, Albury, Australia
}

The Potential Unlimited Programme commenced in 1998, as a collabora1 tive approach to rehabilitation between Southern Area Brain Injury Service in New South Wales and Outward Bound Australia. To date, five programs have seen over 40 participants with acquired brain injury undertake a three stage process including a 7 to 9 day course of outdoor experiential education, imbedded within extensive pre and post-course group work. This review examines two separate pilot evaluations (Walker et al., 2005, Brain Injury, 19, 1237-1241; Thomas, 2004, Brain Injury, 18, 1271-1286) and provides an outline of directions for further program development and research. While there were limitations in the designs employed in these preliminary studies, each indicated participants had likely benefited on important aspects of psychosocial functioning from their involvement. Directions for future research and development include an exciting adaptation of the program for adolescents, which is currently in progress. Our plan to conduct a randomised wait-list controlled trial and further investigation of specific components of the program are outlined. 